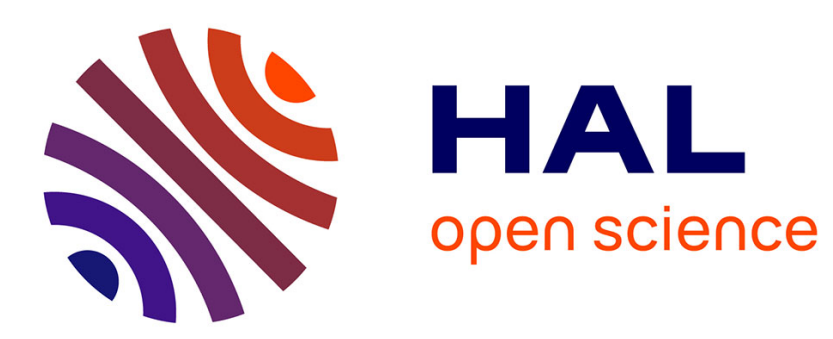

\title{
Fruitiers connus et cultivés en Grèce du Néolithique à l'époque romaine. Confrontation des données archéobotaniques et des sources écrites
}

Clémence Pagnoux

\section{To cite this version:}

Clémence Pagnoux. Fruitiers connus et cultivés en Grèce du Néolithique à l'époque romaine. Confrontation des données archéobotaniques et des sources écrites. Archeosciences, revue d'Archéométrie, 2019, 43, pp.27-52. 10.4000/archeosciences.6134 . hal-02886332

\section{HAL Id: hal-02886332 \\ https://hal.science/hal-02886332}

Submitted on 4 Dec 2020

HAL is a multi-disciplinary open access archive for the deposit and dissemination of scientific research documents, whether they are published or not. The documents may come from teaching and research institutions in France or abroad, or from public or private research centers.
L'archive ouverte pluridisciplinaire HAL, est destinée au dépôt et à la diffusion de documents scientifiques de niveau recherche, publiés ou non, émanant des établissements d'enseignement et de recherche français ou étrangers, des laboratoires publics ou privés. 


\title{
Fruitiers connus et cultivés en Grèce du Néolithique à l'époque romaine - Confrontation des données archéobotaniques et des sources écrites
}

\author{
Cultivated Fruit Trees in Greece from Neolithic to Roman Time - \\ Comparison of Archaeobotanical Data and Written Sources
}

\author{
Clémence Pagnoux ${ }^{\mathrm{a}}$
}

Résumé : L'histoire de l'exploitation des arbres fruitiers en Grèce est encore mal connue et seuls la vigne et l'olivier ont bénéficié d'un intérêt de longue date. L'objectif de cet article est de mettre en lumière l'évolution de la diversité fruitière connue et exploitée en Grèce du Néolithique à l'époque romaine, mais également le processus d'émergence de l'arboriculture. Pour ce faire, le matériel carpologique (restes de graines et de fruits) publié de 68 sites a été pris en compte dans une synthèse. Les mentions de fruits et de fruitiers ont été inventoriées dans les documents épigraphiques en grec mycénien et classique, ainsi que dans les textes d'auteurs antiques. La confrontation de ces sources révèle une évolution du cortège des fruitiers utilisés : vigne, olivier et figuier dominent à toutes les périodes, l'importance de certains fruits sauvages (ronce, sureau, cornouiller) décroît après l'âge du Bronze et de nouveaux fruits sont alors introduits (grenade, noix, pêche, coing par exemple). Les premières formes d'arboriculture que sont les haies, les lisières et l'entretien des arbres dans leur milieu naturel sont complétées, au Bronze récent, par des plantations de fruitiers. De grands vignobles apparaissent à l'époque classique, tandis que la culture de certains fruitiers d'intérêt économique moindre reste limitée à de moins nombreux individus, parfois relégués aux zones marginales de l'espace domestique.

\begin{abstract}
Little is known concerning the history of arboriculture in Greece; only the grapevine and the olive tree have been a subject of interest for a long time. The aim of this paper is to shed light on the diversity of cultivated fruit trees in Greece between the Neolithic and the Roman period. This is why published archaeobotanical data (seeds and fruits) from 68 sites were taken into account in our synthesis. A survey of all references to fruits and fruit trees in epigraphic documents (Mycenaean and classic Greek) and in ancient authors has also been achieved. Our approach reveals how fruit trees were used from the Neolithic up to the Roman period; while the grapevine, the olive tree and the fig tree predominate all the time, it is clear that the importance of certain wild fruits (blackberry, elder, cornelian cherry) decreases after the Bronze Age as new others are introduced (pomegranate, walnut, peach, quince for example). The first manifestations of arboriculture concern woodland edges and partially cleared land plots, real fruit tree plantations appear during the late Bronze Age, at the latest. Extensive vineyards appear during the Classical period, while fewer fruit trees of lesser economic interest are perhaps cultivated on the marginal areas of the domestic space.
\end{abstract}

Mots clés : fructiculture, archéobotanique, épigraphie, Grèce ancienne, littérature grecque, Protohistoire

Keywords: fruit cultivation, archaeobotany, epigraphy, ancient Greece, Greek literature, Prehistory

LIRA Laboratory, Department of Archaeology, School of History and Archaeology, University of Thessaloniki, 54124 Thessaloniki, Grèce - -Center for Interdisciplinary Research and Innovation (CIRI-AUTH), Balkan Center, Buildings A \& B. Thessaloniki, 10th km Thessaloniki-Thermi Rd, P.O. Box 8318, 57001, Grèce.(Clemence.pagnoux@orange.fr) 


\section{${ }^{\mathrm{A}} \mathbf{1}$. INTRODUCTION}

L'arboriculture fruitière en Grèce ancienne a longtemps été identifiée, dans l'imaginaire des archéologues, des historiens et de leurs lecteurs, à la viticulture et à l'oléiculture, composantes des sociétés hiérarchisées qui se développent en Crète et dans le Péloponnèse au Bronze récent (Renfrew, 1972; Gilman, 1981; Halstead, 1992). Viticulture et oléiculture sont ainsi devenues les deux symboles de la fructiculture en Grèce, mais également deux composantes essentielles de la culture mycénienne puis de la culture grecque classique.

L'essor des études paléoenvironnementales et la prise en compte des restes végétaux fossiles lors des fouilles archéologiques ont permis d'apporter de nouveaux éléments sur la présence des arbres fruitiers dans l'environnement des sites et sur la place des fruits dans l'alimentation des sociétés passées. A partir des années 1960, les premières études carpologiques se développent en Grèce (Hopf, 1961, 1962; Renfrew, 1966, 1969; Renfrew, 1972). Les sites préhistoriques ont les premiers bénéficié de ces études, ainsi la question de la culture des fruitiers, et en particulier celle de la vigne, s'est posée pour des périodes bien antérieures au $2^{\mathrm{e}}$ millénaire avant notre ère, date supposée d'introduction de la viticulture en Grèce. Les découvertes de restes de pressage (pépins de raisin, fragments de peau et de rafle carbonisés) mis au jour à Myrtos en Crète (Bronze ancien, 2300/2200 avant notre ère, Renfrew, 1972) et à Dikili Tash en Macédoine (Néolithique récent, 4500-4000 avant notre ère, Valamoti et al., 2007, Valamoti et al., 2015) ont notamment mis au premier plan la question de l'ancienneté de la viticulture en Grèce.

Si la viticulture et l'oléiculture ont fait l'objet de nombreux travaux, fondés sur les sources littéraires, archéologiques et archéobotaniques (e.g. Amouretti 1986; Amouretti et Brun 1993; Blitzer 1993, Hamilakis 1996; Brun 2004), les autres fruitiers ont bénéficié de moins d'intérêt. La synthèse de F. Megaloudi sur les restes de fruits (Megaloudi 2016) est la première qui y soit spécifiquement consacrée, tandis qu'un article de $S$. Amigues propose la première synthèse sur les fruitiers dans la littérature grecque (Amigues 2016).

L'objectif de cet article est de définir les fruitiers exploités et les fruits consommés en Grèce du Néolithique ( $7^{\mathrm{e}}$ millénaire avant notre ère) à l'époque romaine ( $\mathrm{II}^{\mathrm{e}}$ siècle de notre ère), de préciser l'évolution de ce cortège et de préciser les pratiques de culture des arbres fruitiers. Il s'agit non seulement de faire l'inventaire des espèces présentes aux différentes périodes et dans les différentes régions de Grèce, mais également de s'interroger sur les espèces mises en culture, celles potentiellement introduites et éventuellement acclimatées au cours de cette longue période. Les données archéobotaniques comme les sources écrites fournissent des éléments permettant de documenter ces questions, cependant toutes présentent des biais inhérents à leur nature, et aucune n'autorise à elle seule la reconstitution de l'arboriculture fruitière.

C'est pourquoi le propos de cet article est de confronter une synthèse des données carpologiques publiées à un inventaire des espèces mentionnées dans les documents épigraphiques et dans les textes littéraires en grec, afin d'émettre des hypothèses sur l'évolution de la diversité fruitière en Grèce. Les données anthracologiques et palynologiques n'ont pas fait l'objet d'une synthèse exhaustive, cependant elles sont utilisées pour discuter les résultats obtenus et replacer les interprétations qui en découlent dans un contexte plus large.

\section{MATÉriel et MÉTHOde}

\section{Définition des fruits}

De précédents travaux ont soulevé la difficulté à définir les fruits et les fruitiers (Ruas, 1996; Ruas et al., 2005; Bouby et Ruas, 2014; Ruas, 2016). Toute définition possible dépasse nécessairement l'acception botanique (transformation d'une fleur après fécondation) de même que la définition culturelle qui place les fruits parmi les mets sucrés en Europe occidentale. Une définition " archéobotanique » des fruits prendrait donc en compte un ensemble de critères à la fois botaniques et culturels. De fait, dans l'étude des restes carpologiques, la catégorie des « fruits » désigne communément ce qui n'est ni céréale, ni légumineuse, ni oléagineux, ni herbacées sauvages et dont les usages peuvent être multiples (Ruas et al., 2005). Cette catégorie est encore subdivisée selon d'autres critères : fruits secs ou charnus, fruits cueillis ou cultivés... Bien que ces catégories, souvent binaires, soient parfois insuffisantes pour classer la diversité à laquelle nous sommes confrontés, cela permet de prendre en compte des fruits comme les glands, les fruits du pistachier térébinthe ou du myrte qui ne rentrent pas dans la catégorie des fruits " traditionnels». Les fruits ont un usage alimentaire, ils peuvent également servir dans l'artisanat (les baies de sureau peuvent être utilisées pour la teinture, par exemple), ils peuvent être consommés frais, séchés ou transformés (huile d'olive et vin pour ne citer que deux produits transformés). De plus, les fruits ont leur place en contexte rituel et religieux, notamment dans la religion grecque antique (Megaloudi, 2005, Megaloudi et al., 2007), mais également en contexte funéraire ou rituel dès le Néolithique (Margaritis, 2014a; Valamoti, 2011). Certains sont chargés d'une signification symbolique forte : la gre- 
nade par exemple, associée à plusieurs divinités grecques, est un symbole de fertilité, d'abondance, de renaissance et d'éternité (Chevalier et Gheerbrant, 1982).

Les fruits retenus dans ce travail recoupent les ensembles considérés par de précédents travaux sur la fructiculture (e.g. Bouby et Ruas, 2014; Ruas et al., 2005; Ruas, 2016; Megaloudi, 2016). Ce sont les productions de plantes pérennes, principalement des ligneux propagés par voie végétative. Ils peuvent être domestiques ou sauvages, cultivés ou cueillis. Enfin, sont pris en compte les fruits classés comme tels par les sources écrites mais que nous ne considérons pas comme des fruits (comme le concombre), ainsi que d'autres espèces que les sources écrites ne traitent pas parmi les fruitiers notamment du fait de leur caractère non ligneux (par exemple la ronce).

\section{Synthèse des données carpologiques}

Les données carpologiques constituent la source principale. La dernière synthèse de ces données pour la Grèce remonte à 2006 (Megaloudi, 2006) et les synthèses régionales comme les études thématiques se développent (Valamoti, 2004, 2009; Livarda et Kotzamani, 2013).

Les restes carpologiques (restes de fruits et graines) publiés de 68 sites datés du Néolithique ancien (6600/6500 avant notre ère) à l'époque romaine (jusqu'au $4^{\mathrm{e}}$ siècle de notre ère) ont fait l'objet d'une synthèse (figure 1, tableau 1). Les restes collectés sur tous ces sites l'ont été selon différentes méthodes. La plupart a fait l'objet de prélèvements de sédiment, ciblés ou systématiques. Cependant, sur neuf sites, les restes ont été ramassés à vue au cours de la fouille par les archéologues. Enfin, pour 14 sites, nous ne disposons d'aucune information sur le mode d'acquisition des restes. Par ailleurs, certains rapports ne présentent pas de données quantifiées : il s'agit généralement de rapports préliminaires, qui devraient être suivis de publications qui fourniront toutes ces informations et permettront peut-être une synthèse plus précise, reposant sur des données plus robustes.

Par ailleurs, ce corpus se caractérise par des déséquilibres géographiques et chronologiques. Les périodes représentées par le plus grand nombre de sites sont le Néolithique récent (de 5500/5300 avant notre ère à 4100/3800; 17 sites), l'âge du Bronze ancien (de 3500/3300 à 2300/2200; 14 sites) et l'âge du Bronze récent (de 1700/1500 à 1100/1050; 16 sites). Les périodes historiques sont nettement moins bien documentées, avec neuf sites pour les époques archaïque et classique, cinq sites pour l'époque hellénistique et un seul pour l'époque romaine (figure 1, tableau 1).

Les régions les mieux explorées d'un point de vue archéobotanique sont la Macédoine (17 sites si l'on y rattache la Thrace, qui ne compte qu'un site), la Thessalie et le Péloponnèse (13 sites dans chaque région). Les îles sont les régions les moins étudiées : la Crète compte 9 sites, les Cyclades en comprennent 5, et les îles du Nord et de l'Est de la mer Egée (Thasos, Lesbos et Samos) ne comptent que quatre sites. Aucune étude n'a encore été publiée sur l'ouest de la Grèce.

Lobservation croisée des caractéristiques chronologiques et géographiques du corpus permet alors de mettre en évidence le fait que, outre ces déséquilibres quantitatifs d'une région à l'autre et d'une période à l'autre, géographie et chronologie sont très liées. En effet, la plupart des sites néo-
Figure 1 : (Voir planche couleur $\bullet$ ) Localisation des sites archéologiques mentionnés dans le texte.

Figure 1: (See colour plate $\bullet \bullet)$ Geographical location of archaeological sites mentioned in the text.

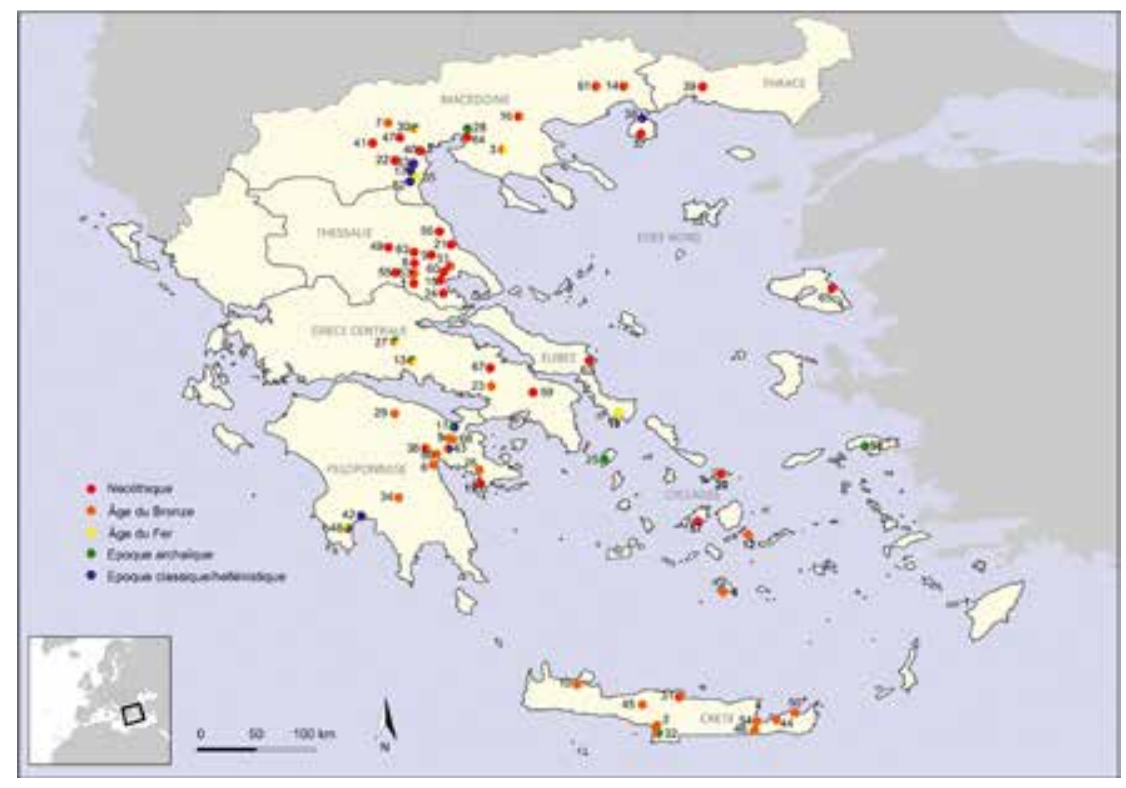




\begin{tabular}{|c|c|c|c|c|c|c|}
\hline Nom du site & Région & Période & Contexte & $\begin{array}{l}\text { Mode d'acquisition } \\
\text { des restes }\end{array}$ & Auteur de l'étude & Références \\
\hline 1. Achilleion & Thessalie & $\begin{array}{l}\text { Néolithique ancien- } \\
\text { Néolithique moyen }\end{array}$ & Habitat & Prélèvements & J. Renfrew & Renfrew 1989 \\
\hline 2. Aghia Triada & Crète & Bronze récent & Palais & Ramassage manuel & M. Follieri & Follieri 1979 \\
\hline 3. Aghios Mamas & Macédoine & $\begin{array}{l}\text { Bronze moyen-âge } \\
\text { du Fer }\end{array}$ & Habitat & $\begin{array}{l}\text { Prélèvements; } \\
0.3 \mathrm{~mm}\end{array}$ & H. Kroll & $\begin{array}{l}\text { Becker et Kroll } \\
\text { 2008, Kroll } 2003\end{array}$ \\
\hline 4. Akrotiri & Cyclades & $\begin{array}{l}\text { Bronze moyen- } \\
\text { Bronze final }\end{array}$ & Habitat & Prélèvements & A. Sarpaki & $\begin{array}{l}\text { Sarpaki 1992, Asouti } \\
\text { 2003, Sarpaki et } \\
\text { Asouti } 2008\end{array}$ \\
\hline 5. Arapi & Thessalie & Néolithique final & Habitat & Non précisé & H. Kroll & Kroll 1981 \\
\hline 6. Argos Aspis & Péloponnèse & Bronze moyen & Habitat & $\begin{array}{l}\text { Prélèvement; } \\
0.3 \mathrm{~mm}\end{array}$ & E. Margaritis & $\begin{array}{l}\text { Philippa-Touchais et } \\
\text { al. } 2014\end{array}$ \\
\hline 7. Archondiko & Macédoine & Bronze ancien & Habitat & $\begin{array}{l}\text { Prélèvements; } \\
0.3 \mathrm{~mm}\end{array}$ & S.M. Valamoti & Valamoti 1997 \\
\hline 8. Argissa Magoula & Thessalie & $\begin{array}{l}\text { Néolithique précéra- } \\
\text { mique-Néolithique } \\
\text { moyen }\end{array}$ & Habitat & Non précisé & M. Hopf & Hopf 1962b \\
\hline 9. Ayia Sotiria & Péloponnèse & Bronze récent & Funéraire & $\begin{array}{l}\text { Prélèvement; } \\
0.3 \mathrm{~mm}\end{array}$ & $\begin{array}{l}\text { A. Livarda, G. Kot- } \\
\text { zamani }\end{array}$ & $\begin{array}{l}\text { Kotzamani et } \\
\text { Livarda } 2017\end{array}$ \\
\hline $\begin{array}{l}\text { 10. Chamalevri- } \\
\text { Tzambakas }\end{array}$ & Crète & Bronze ancien & & Non précisé & A. Sarpaki & Sarpaki 1999 \\
\hline 11. Corinthe & Péloponnèse & $\begin{array}{l}\text { Epoques archaïque } \\
\text { et classique }\end{array}$ & Sanctuaire & Prélèvements; $1 \mathrm{~mm}$ & J. Hansen & Bookidis et al. 1999 \\
\hline 12. Dhaskalio Keros & Cyclades & Bronze ancien & Habitat & $\begin{array}{l}\text { Prélèvements; } \\
0.3 \mathrm{~mm}\end{array}$ & E. Margaritis & Margaritis 2013b \\
\hline $\begin{array}{l}\text { 13. Delphes pilier } \\
\text { des Rhodiens }\end{array}$ & Grèce centrale & $\begin{array}{l}\text { Bronze récent- } \\
\text { époque archaïque }\end{array}$ & Habitat et rituel & $\begin{array}{l}\text { Prélèvements; } \\
0.5 \mathrm{~mm}\end{array}$ & P. Marinval & $\begin{array}{l}\text { Luce et Marinval } \\
2008\end{array}$ \\
\hline 14. Dikili Tash & Macédoine & $\begin{array}{l}\text { Néolithique final- } \\
\text { Bronze ancien }\end{array}$ & Habitat & $\begin{array}{l}\text { Prélèvements : } \\
0.5 \mathrm{~mm}\end{array}$ & S.M. Valamoti & Valamoti 2004 \\
\hline 15. Dimini & Thessalie & Néolithique final & Habitat & Non précisé & H. Kroll & Kroll 1979b \\
\hline 16. Dimitra & Macédoine & $\begin{array}{l}\text { Néolithique final- } \\
\text { Bronze récent }\end{array}$ & Habitat & $\begin{array}{l}\text { Prélèvements : } \\
0.75 \mathrm{~mm}\end{array}$ & J. Renfrew & Renfrew 1997 \\
\hline 17. Douvari & Macédoine & Epoque hellénistique & Habitat rural & $\begin{array}{l}\text { Prélèvements; } \\
0.3 \mathrm{~mm}\end{array}$ & E. Margaritis & $\begin{array}{l}\text { Margaritis 2015, } \\
2016\end{array}$ \\
\hline $\begin{array}{l}\text { 18. Erétrie, sanc- } \\
\text { tuaire d'Apollon }\end{array}$ & Eubée & Âge du fer & Sanctuaire & $\begin{array}{l}\text { Prélèvements; } \\
0.3 \mathrm{~mm}\end{array}$ & E. Margaritis & Margaritis 2013a \\
\hline 19. Franchthi & Péloponnèse & $\begin{array}{l}\text { Néolithique ancien } \\
\text { et moyen }\end{array}$ & Habitat (grotte) & $\begin{array}{l}\text { Prélèvements; } \\
1.5 \mathrm{~mm}\end{array}$ & J. Hansen & Hansen 1991 \\
\hline 20. Ftelia & Cyclades & Néolithique final & Habitat & $\begin{array}{l}\text { Prélèvements; } \\
0.5 \mathrm{~mm}\end{array}$ & $\begin{array}{l}\text { P. Marinval, F. } \\
\text { Megaloudi }\end{array}$ & $\begin{array}{l}\text { Megaloudi et Marin- } \\
\text { val } 2002\end{array}$ \\
\hline 21. Ghediki & Thessalie & $\begin{array}{l}\text { Néolithique précéra- } \\
\text { mique }\end{array}$ & Habitat & Non précisé & J. Renfrew & Renfrew 1966 \\
\hline 22. Giannitsa B & Macédoine & Néolithique ancien & Habitat & $\begin{array}{l}\text { Prélèvements; } \\
0.3 \mathrm{~mm}\end{array}$ & S.M. Valamoti & Valamoti 1995 \\
\hline 23. Gla & Grèce centrale & Bronze récent & Site de hauteur & Non précisé & G. Jones & Jones 1995 \\
\hline \multirow{2}{*}{ 24. Iolkos } & \multirow{2}{*}{ Thessalie } & Âge du Bronze & \multirow{2}{*}{ Habitat } & \multirow{2}{*}{ Non précisé } & \multirow{2}{*}{ G. Jones, J. Renfrew } & Renfrew 1966 \\
\hline & & Âge du Fer & & & & \begin{tabular}{|l|} 
Jones 1982 \\
\end{tabular} \\
\hline 25. Ipsili & Cyclades & Epoque archaïque & Habitat & $\begin{array}{l}\text { Contenu de réci- } \\
\text { pients }\end{array}$ & F. Megaloudi & Megaloudi 2006 \\
\hline 26. Iria & Péloponnèse & Bronze final & Habitat & Ramassage manuel & U. Willerding & Willerding 1973 \\
\hline 27. Kalapodi & Grèce centrale & $\begin{array}{l}\text { Bronze final - } \\
\text { Époque classique }\end{array}$ & Sanctuaire & Prélèvements & H. Kroll & Kroll 1993 \\
\hline 28. Karambournaki & Macédoine & Époque archaïque & Habitat & $\begin{array}{l}\text { Prélèvements; } \\
0.3 \mathrm{~mm}\end{array}$ & S.M. Valamoti & Valamoti 2005 \\
\hline
\end{tabular}




\begin{tabular}{|c|c|c|c|c|c|c|}
\hline 29. Kassaneva & Péloponnèse & Bronze ancien & Habitat & Prélèvements & G. Fiorentino & Colaianni et al. 2012 \\
\hline 30. Kastanas & Macédoine & $\begin{array}{l}\text { Bronze final - } \\
\text { époque classique }\end{array}$ & Habitat & $\begin{array}{l}\text { Prélèvements; } \\
0.2 \mathrm{~mm}\end{array}$ & H. Kroll & $\begin{array}{l}\text { Kroll 1979a, Kroll } \\
1983\end{array}$ \\
\hline 31. Knossos & Crète & $\begin{array}{l}\text { Néolithique ancien- } \\
\text { néolithique récent }\end{array}$ & Habitat & Prélèvements & A. Sarpaki & $\begin{array}{l}\text { Sarpaki 2013, Badal } \\
\text { et Ntinou } 2013\end{array}$ \\
\hline $\begin{array}{l}\text { 31. Knossos "Unex- } \\
\text { plored Mansion" }\end{array}$ & Crète & Bronze récent & Habitat & Ramassage manuel & G. Jones & Jones 1984 \\
\hline 32. Kommos & Crète & $\begin{array}{l}\text { Bronze moyen- } \\
\text { époque archaïque }\end{array}$ & Habitat & Ramassage manuel & C.T. Shay, J.M Shay & $\begin{array}{l}\text { Shay et Shay 2000, } \\
\text { Shay et al. } 1995\end{array}$ \\
\hline 33. Kompoloi & Macédoine & Epoque hellénistique & Habitat rural & $\begin{array}{l}\text { Prélèvements; } 0.3 \\
\mathrm{~mm}\end{array}$ & E. Margaritis & $\begin{array}{l}\text { Margaritis 2015, } \\
2016\end{array}$ \\
\hline 34. Kouphovouno & Péloponnèse & Bronze moyen & Habitat & Prélèvements & F. Megaloudi & Megaloudi 2006 \\
\hline 35. Krania & Macédoine & $\begin{array}{l}\text { Âge du Fer; hellé- } \\
\text { nistique }\end{array}$ & Habitat & $\begin{array}{l}\text { Prélèvements; } \\
0.25 \mathrm{~mm}\end{array}$ & E. Margaritis & $\begin{array}{l}\text { Margaritis 2002b, } \\
\text { Margaritis 2014b, } \\
2015\end{array}$ \\
\hline 36. Lerne & Péloponnèse & $\begin{array}{l}\text { Néolithique final- } \\
\text { Bronze final }\end{array}$ & Habitat & Ramassage manuel & M. Hopf & Hopf 1961, 1962a \\
\hline 37. Limenaria & Macédoine & Néolitique moyen & Habitat & Prélèvements & F. Megaloudi & $\begin{array}{l}\text { Megaloudi 2006, } \\
\text { Megaloudi et Papa- } \\
\text { dopoulos } 2012\end{array}$ \\
\hline 38. Limenas & Macédoine & Époque classique & Funéraire & $\begin{array}{l}\text { Prélèvements; } \\
0.3 \mathrm{~mm}\end{array}$ & F. Megaloudi & $\begin{array}{l}\text { Megaloudi et al. } \\
2007\end{array}$ \\
\hline 39. Makri & Thrace & Néolithique final & Habitat & $\begin{array}{l}\text { Prélèvements; } \\
0.3 \mathrm{~mm}\end{array}$ & S.M. Valamoti & Valamoti 2004 \\
\hline 40. Makriyalos & Macédoine & Néolithique final & Habitat & $\begin{array}{l}\text { Prélèvements; } \\
0.3 \mathrm{~mm}\end{array}$ & S.M. Valamoti & \begin{tabular}{|l} 
Valamoti 1999, \\
Valamoti 2004
\end{tabular} \\
\hline 41. Mavropigi & Macédoine & Néolithique ancien & Habitat et funéraire & \begin{tabular}{|l|} 
Prélève- \\
ment; $0.3 \mathrm{~mm}$
\end{tabular} & S.M. Valamoti & Valamoti 2011 \\
\hline 42. Messène & Péloponnnèse & Epoque hellénistique & Rituel & Ramassage manuel & F. Megaloudi & Megaloudi 2005 \\
\hline 43. Midea & Péloponnèse & $\begin{array}{l}\text { Bronze récent; } \\
\text { époque romaine }\end{array}$ & Site de Hauteur & Non précisé & C.T. Shay, J.M Shay & Shay et al. 1998 \\
\hline 44. Mochlos & Crète & Bronze récent & Habitat & Prélèvements & A. Sarpaki & $\begin{array}{l}\text { Soles 2003, Sarpaki } \\
\text { et Bending } 2004\end{array}$ \\
\hline 45. Monastiraki & Crète & Bronze moyen & Habitat & $\begin{array}{l}\text { Prélèvements; } 0.25 \\
\mathrm{~mm}\end{array}$ & $\begin{array}{l}\text { G. Fiorentino, A. } \\
\text { Sarpaki, F. Solinas }\end{array}$ & $\begin{array}{l}\text { Fiorentino et Solinas } \\
\text { 2006; Sarpaki } 2012\end{array}$ \\
\hline 46. Myrtos & Crète & Bronze ancien & Habitat & Prélèvements & J. Renfew & Renfrew 1972 \\
\hline 47. Nea Nikomedeia & Macédoine & Néolithique ancien & Habitat & Ramassage manuel & W. Van Zeist & $\begin{array}{l}\text { Van Zeist et Bot- } \\
\text { tema } 1971\end{array}$ \\
\hline 48. Nichoria & Péloponnèse & $\begin{array}{l}\text { Bronze moyen- } \\
\text { époque classique }\end{array}$ & Site de hauteur & Prélèvements & C.T. Shay, J.M Shay & Shay et Shay 1978 \\
\hline 49. Otzaki & Thessalie & $\begin{array}{l}\text { Néolithique ancien- } \\
\text { Néolithique moyen }\end{array}$ & Habitat & Non précisé & H. Kroll & Kroll 1981 \\
\hline 50. Papadiokampos & Crète & Bronze récent & Habitat & Non précisé & E. Margaritis & Brogan et al. 2013 \\
\hline 51. Pefkakia & Thessalie & $\begin{array}{l}\text { Néolithique final- } \\
\text { Bronze moyen }\end{array}$ & Habitat & Non précisé & H. Kroll & Kroll 1981 \\
\hline 52. Platania & Macédoine & Epoque hellénistique & Habitat rural & $\begin{array}{l}\text { Prélèvements; } \\
0.3 \mathrm{~mm}\end{array}$ & E. Margaritis & $\begin{array}{l}\text { Margaritis 2015, } \\
2016\end{array}$ \\
\hline $\begin{array}{l}\text { 53. Plateia Magoula } \\
\text { Zarkou }\end{array}$ & Thessalie & $\begin{array}{l}\text { Néolithique moyen- } \\
\text { Bronze moyen }\end{array}$ & Habitat & Ramassage manuel & G. Jones & $\begin{array}{l}\text { Jones et Halstead } \\
1993\end{array}$ \\
\hline $\begin{array}{l}\text { 54. Priniatikos } \\
\text { Pyrgos }\end{array}$ & Crète & Bronze ancien & Habitat & $\begin{array}{l}\text { Prélèvements; } \\
0.5 \mathrm{~mm}\end{array}$ & G. Kotzamani & Molloy et al., 2014 \\
\hline 55. Prodromos & Thessalie & Néolithique ancien & Habitat & Ramassage manuel & G. Jones & $\begin{array}{l}\text { Halstead et Jones } \\
1980\end{array}$ \\
\hline 56. Rachmani & Thessalie & Néolithique final & Habitat & Non précisé & J. Renfrew & Renfrew 1966 \\
\hline 57. Saliagos & Cyclades & Néolithique final & Habitat & Non précisé & J. Renfrew & Renfrew 1968 \\
\hline
\end{tabular}




\begin{tabular}{|c|c|c|c|c|c|c|}
\hline 58. Samos Heraion & Egée nord & Epoque archaïque & Sanctuaire & $\begin{array}{l}\text { Prélèvements; } \\
0.2 \mathrm{~mm}\end{array}$ & D. Kučan & Kučan 1995, 2000 \\
\hline 59. Sarakenos & Grèce centrale & Néolithique final & Habitat (grotte) & Prélèvements & F. Megaloudi & Megaloudi 2008 \\
\hline 60. Sesklo & Thessalie & $\begin{array}{l}\text { Néolithique précéra- } \\
\text { mique-Néolihitque } \\
\text { final }\end{array}$ & Habitat & Non précisé & H. Kroll, J. Renfrew & $\begin{array}{l}\text { Renfrew 1966, Krol } \\
1981\end{array}$ \\
\hline 61. Sitagroi & Macédoine & $\begin{array}{l}\text { Néolithique moyen- } \\
\text { Bronze ancien }\end{array}$ & Habitat & $\begin{array}{l}\text { Prélèvements; } \\
1.5 \mathrm{~mm}\end{array}$ & J. Renfrew & Renfrew 2003 \\
\hline $\begin{array}{l}\text { 62. Skoteini Thar- } \\
\text { rounia }\end{array}$ & Eubée & $\begin{array}{l}\text { Néolithique final- } \\
\text { Bronze ancien }\end{array}$ & Habitat & Prélèvements & M. Mangafa & Mangafa 1993 \\
\hline 63. Soufli & Thessalie & $\begin{array}{l}\text { Néolithique } \\
\text { précéram.-Néoli- } \\
\text { thique ancien }\end{array}$ & Habitat & Non précisé & J. Renfrew & Renfrew 1966 \\
\hline 64. Stavroupoli & Macédoine & Néolithique moyen & Habitat & $\begin{array}{l}\text { Prélèvements; } \\
0.25 \mathrm{~mm}\end{array}$ & E. Margaritis & $\begin{array}{l}\text { Margaritis 2002, } \\
\text { Margaritis 2004 }\end{array}$ \\
\hline 65. Thermi B & Egée nord & Néolithique final & Habitat & $\begin{array}{l}\text { Prélèvements; } \\
0.3 \mathrm{~mm}\end{array}$ & S.M. Valamoti & Valamoti 1992 \\
\hline 66. Tirynthe & Peloponnèse & $\begin{array}{l}\text { Bronze ancien- } \\
\text { Bronze final }\end{array}$ & Palais & $\begin{array}{l}\text { Prélèvements; } \\
0.2 \mathrm{~mm}\end{array}$ & H. Kroll & Kroll 1982, 1984 \\
\hline $\begin{array}{l}\text { 67. Toumba Balo- } \\
\text { menou }\end{array}$ & Grèce centrale & $\begin{array}{l}\text { Néolithique ancien- } \\
\text { Néolithique moyen }\end{array}$ & Habitat & $\begin{array}{l}\text { Prélèvements; } \\
0.3 \mathrm{~mm}\end{array}$ & A. Sarpaki & Sarpaki 1995 \\
\hline 68. Tsoungiza & Péloponnèse & $\begin{array}{l}\text { Néolithique final- } \\
\text { Bronze récent }\end{array}$ & Habitat & Prélèvements; $1 \mathrm{~mm}$ & S. Allens, J. Hansen & $\begin{array}{l}\text { Hansen et Allen } \\
2011\end{array}$ \\
\hline
\end{tabular}

Tableau 1 : Liste et références des sites archéologiques mentionnés dans le texte.

Table 1: List and references of archaeological sites mentioned in the text.

lithiques qui ont fait l'objet d'études archéobotaniques se trouvent en Macédoine et en Thessalie, car cette période a été largement étudiée dans ces régions, notamment suite à la découverte des grands sites de Dimini et Sesklo en Thessalie (Tsoundas, 1908). En Crète, dans le Péloponnèse et en Grèce centrale où peu de sites néolithiques ont été étudiés, ce sont surtout des phases de l'âge du Bronze qui sont bien connues. Cela s'explique par l'intérêt soulevé par les périodes minoenne et mycénienne dans ces régions, qui ont longtemps orienté les recherches archéologiques. Enfin, les assemblages carpologiques d'époque archaïque, classique et hellénistique proviennent pour la plupart de Macédoine, de Grèce centrale et du Péloponnèse, où les recherches archéologiques sont globalement plus développées que dans les îles.

D'autre part, outre le fait que les habitats soient en nombre bien plus important que les autres types de sites (sanctuaires, ensemble funéraire, site palatiaux), la répartition des types de sites est également très liée à la géographie et à la chronologie. En effet, les sites de Thessalie et du Nord sont surtout des habitats, et on ne trouve de sanctuaire que dans le sud de la Grèce (Péloponnèse, Crète et Grèce centrale), ainsi qu'à Samos (temple d'Héra), et tous sont postérieurs à la fin de l'âge du Bronze. Enfin, il n'y a de sites de type "palais » que pour l'âge du Bronze, dans le Péloponnèse, en Grèce centrale, et en Crète, où se sont développées les sociétés palatiales mycéniennes et minoenne. En outre et sans corrélation géographique ni chronologique, le corpus des sites comprend deux ensembles rituels (les dépôts sacrificiels de Delphes, $8^{\mathrm{e}}-7^{\mathrm{e}} \mathrm{s}$. avant notre ère, Luce et Marinval, 2008, et les dépôts rituels de Messène, $3^{\mathrm{e}}$ s. avant notre ère, Megaloudi, 2005), et deux ensembles funéraires, l'un daté du Néolithique ancien à Mavropigi, Macédoine (Valamoti, 2011), l'autre d'époque classique, situé à Liménas sur l'île de Thasos (Megaloudi et al., 2007).

Compte tenu des déséquilibres chronologiques et géographiques observés, des regroupements de phases chronologiques et de régions ont été effectués afin de limiter les disparités numériques d'un ensemble à l'autre. Ainsi les phases du Néolithique ancien et du Néolithique moyen sont regroupées, de même que celles de l'âge du Bronze ancien et moyen. Le seul site de Thrace (Makri) est regroupé avec ceux de Macédoine, de même que ceux des îles de Thasos et Lesbos (nord de la mer Egée). Les quelques sites de l'île d'Eubée sont regroupés avec ceux de Grèce centrale. Les sites des Cyclades sont peu nombreux, mais ils ne sauraient former un ensemble cohérent ni avec le Péloponnèse ni avec la Crète, c'est pourquoi ils sont conservés sous la forme d'un groupe autonome.

La très grande majorité des restes carpologiques provenant de Grèce est conservée sous forme carbonisée. Cette grande homogénéité de mode de conservation permet la comparaison des sites. Toutefois on peut regretter la très 
faible quantité de contextes humides, qui auraient pu livrer davantage de restes de fruits. Un seul ensemble imbibé a pu être intégré à ce travail et provient du sanctuaire archaïque d'Héra (Heraion) à Samos (viII siècle). Il s'agit d'un bassin au contact de la nappe phréatique, à proximité de la mer. Les restes minéralisés ne représentent qu'une petite partie des restes carpologiques provenant de sites archéologiques grecs et ne documentent aucun taxon qui ne se trouve pas déjà attesté par les ensembles carbonisés.

Seuls les ensembles carbonisés sont pris en compte dans les analyses. Les données issues de l'Heraion de Samos seront toutefois exposées à la suite des résultats des analyses et prises en compte dans la discussion.

Afin de pouvoir faire porter l'analyse sur l'ensemble du corpus, y compris sur les sites pour lesquels aucune donnée quantifiée n'était disponible, il a été décidé de comparer les occupations par l'ubiquité : la présence ou l'absence des différents taxons est donnée pour chaque occupation. L'objectif de cette analyse est de caractériser l'évolution chronologique des assemblages et leur distribution géographique. À partir des données codées en présence/absence, on a pu établir le nombre d'occurrences de chaque taxon, c'est-à-dire le nombre de phases d'occupation où il est présent. Celui-ci a été calculé par période et par région. Les sites ont été traités par phases chronologiques telles qu'elles étaient définies dans les publications.

Afin de ne pas multiplier les taxons, et pour ne pas donner un poids trop important aux biais induits par des états de conservation différents qui ont pu conduire à des identifications moins précises, les taxons ont été regroupés. Le regroupement de taxons vise à rassembler sous un même nom tous ceux qui désignent très probablement la même chose (Colledge, 2001; Valamoti, 2004). Par exemple, Vitis cf. vinifera et Vitis vinifera ont été regroupés. Dans le cas du sureau, les restes ont tous été attribués au taxon Sambucus, qui regroupe $S$. nigra, S. racemosa, S. nigralracemosa, Sambucus ebulus et Sambucus sp., car tous ces taxons sont attestés avec une fréquence proche. Bien que les fruits du sureau yièble (S. ebulus) soient toxiques lorsqu'ils sont consommés frais (Couplan, 1989; Lieutaghi, 2004), les restes de ce taxon ont été pris en compte dans les analyses. En effet, d'une part, les ensembles identifiés comme Sambucus sp. peuvent en contenir, d'autre part, ils peuvent être utilisés en médecine ou en teinture de même que les fruits du sureau noir (Lieutaghi, 2004).

Ainsi, 111 ensembles datés appartenant à 67 sites et 24 taxons ont été exploités dans ces analyses.

\section{Épigraphie}

Aux résultats obtenus par les analyses du matériel carpologique ont été confrontées les mentions de fruits et de fruitiers dans les documents épigraphiques en grec mycénien et en grec classique.

Les tablettes en linéaire $B$ constituent les plus anciens documents écrits et déchiffrés découverts en Grèce continentale et en Crète. Il s'agit de listes, de comptes et d'inventaires de magasins, de listes de produits contrôlés ou distribués, de denrées impliquées dans la fabrication de produits soumis au contrôle d'une autorité centrale, le palais (e.g. Poursat in Treuil et al., 2008). Ces listes étaient gravées sur des tablettes d'argile, dont certaines nous sont parvenues grâce à une cuisson accidentelle lors des incendies des palais à la fin du XIII ${ }^{\mathrm{e}}$ siècle avant notre ère.

La lecture des différents ouvrages ou articles thématiques (Bennett, 1958; Chadwick, 1976; Melena, 1983; Palmer, 1994, 1996; Killen, 1999, 2004; Bendall, 2007 ; Rougemont, 2008; Fappas, 2010), ainsi que celle de certains corpus d'édition des textes mycéniens (Ventris et Chadwick, 1973; Bennett et Olivier, 1973, 1976; Killen et Olivier, 1989), a permis d'établir une liste de 273 documents susceptibles de fournir des informations sur les fruitiers. Aucune nouvelle édition ni nouvelle traduction de ces textes n'est proposée dans cet article. Tous les documents utilisés dans cette étude sont datées du XIII ${ }^{\mathrm{e}}$ siècle avant notre ère. Il convient de rappeler que les tablettes provenant des palais mycéniens documentent les activités dans lesquelles l'autorité palatiale intervient, et ne reflètent donc pas le régime alimentaire du commun des habitants du Péloponnèse et de Crète à l'âge du Bronze. Cependant, elles attestent l'exploitation de certains fruitiers et la consommation des fruits qu'ils produisent, sans pour autant que puisse être exclu le recours à d'autres fruits qui n'ont jamais fait l'objet de gestion centralisée (Halstead, 1999, 2011). Ces documents permettent également d'affirmer le rôle social et économique de ces fruits dans le cadre des échanges contrôlés par le palais.

Bien plus tardifs, d'une toute autre nature, les baux ruraux d'époque classique et hellénistique présentent la particularité d'être les seuls témoignages écrits sur l'agriculture de leur temps. Il s'agit de contrats de location entre un bailleur public (sanctuaire, cité ou subdivision du corps civique) et un locataire privé, qui contiennent des clauses relatives à l'entretien du terrain. La plantation ou le renouvellement des arbres fruitiers peuvent ainsi être mentionnés, ainsi que le nombre d'arbres à planter ou à rendre à la fin du bail. Ces textes représentent moins de 300 documents sur l'ensemble des inscriptions de langue grecque. Parmi ces documents, ceux qui abordent les cultures pratiquées sur les terrains mis 
en location sont relativement rares. Le corpus de textes retenus pour ce travail a été établi en s'appuyant sur la synthèse d'I. Pernin sur les baux ruraux de Grèce ancienne (Pernin, 2014). Le corpus utilisé ici comprend des textes de Grèce continentale, des Cyclades, des îles de l'est de l'Egée et de la côte d'Asie Mineure. Un total de 59 documents, équivalant à 84 contrats mentionne explicitement des fruitiers. Ils sont datés du $v^{\mathrm{e}}$ siècle avant notre ère au $\mathrm{II}^{\mathrm{e}}$ siècle de notre ère.

\section{Textes littéraires}

Les textes littéraires constituent la dernière source exploitée pour étudier les fruits connus et consommés en Grèce ancienne. Le choix des textes retenus pour cette étude vise à étudier les descriptions de fruits et de leurs usages, ainsi que les recommandations qui concernent leur culture. En effet, entreprendre un inventaire complet des mentions de fruits et fruitiers dans la littérature grecque, compter toutes les occurrences de chaque espèce et associer chacune à un contexte présenteraient un intérêt certain, toutefois cela constituerait une étude à part entière. En outre, les travaux de $S$. Amigues et en particulier un article consacré aux fruits et aux fruitiers paru en 2016 (Amigues, 2016) propose déjà un inventaire commenté de plusieurs auteurs, notamment Homère. Ainsi les textes investigués ici ne représentent pas un corpus exhaustif des mentions de fruits et de fruitiers dans la littérature grecque, loin s'en faut. L'ensemble des passages relevés a fait l'objet d'une traduction personnelle, sauf lorsque le recours à une autre traduction est signalé. Pour les textes de Théophraste, la traduction de S. Amigues a été utilisée, parfois légèrement remaniée (Amigues, 2003a et $b$; Amigues, 2012).

La source la plus complète sur les plantes connues des Grecs de l'époque classique est l'ouvrage de Théophraste, Recherches sur les plantes, datant du IV siècle avant notre ère. L'inventaire peut être complété par l'ouvrage du médecin Dioscoride ( $\mathrm{I}^{\text {er }}$ siècle de notre ère). Sa Pharmacopée renseigne sur l'usage des fruits en médecine, en particulier le livre 1 qui rassemble les propriétés des arbres, et le livre 5, entièrement consacré à la vigne et au vin. Cet ouvrage fournit plusieurs descriptions d'arbres et de fruits, ainsi que de leurs propriétés.

Outre ces deux ouvrages, les écrits d'Athénée $\left(2^{\mathrm{e}}-3^{\mathrm{e}}\right.$ siècles) permettent de compléter la connaissance des fruits en Grèce. Son ouvrage (Le banquet des philosophes, ou Les Deipnosophistes) prend la forme d'un dialogue entre philosophes qui dissertent sur ce qui se mange et se boit. Athénée rassemble dans ce texte les descriptions, récits et mentions relatives aux aliments et boissons qui se trouvent chez les auteurs grecs qui l'ont précédé. Ainsi, il contient de nom- breux renseignements sur les fruits : des descriptions à travers la littérature grecque, des réflexions sur l'identification de certains fruits d'après les noms ou les descriptions qu'en font les auteurs, de possibles usages des fruitiers d'après l'ensemble des sources examinées.

Si les source littéraires documentent la connaissance, la place, les usages et la perception des fruitiers, elles ne peuvent être utilisés comme des témoignages directs sur leur époque : les choix de leurs auteurs, les pratiques d'écriture et notamment les reprises d'un auteur à l'autre sans citation explicite de ces emprunts, mais aussi l'état de leurs connaissances et le filtre de leur perception du monde végétal sont autant de biais qui donnent une image déformée de la réalité passée. Ces textes contiennent un état des connaissances scientifiques et techniques de leur époque, toutefois certains reflètent surtout les connaissances que leurs auteurs ont de leurs prédécesseurs. De plus, si ces reprises d'un auteur à l'autre sont parfois très fidèles voire identiques au mot près, il arrive également qu'elles soient approximatives et erronées, ce qui produit des informations non pas sur une réalité observable, mais sur la compréhension que les auteurs ont de ceux qui ont écrits avant eux.

Par ailleurs, les classifications des anciens, qu'il s'agisse de classer les fruits et fruitiers dans un traité de médecine ou dans un ouvrage de botanique, ne correspondent pas à notre classification taxinomique. Il est donc difficile d'identifier tous les fruits ou arbres mentionnés à des taxons relevant des classifications actuelles. Les termes génos ( $\gamma \varepsilon \dot{v} v o \varsigma$ ) et eídos

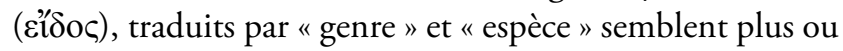
moins interchangeables chez Théophraste (Amigues, 1999; 2003b), et Dioscoride utilise davantage le mot eidos, qui lui permet d'énumérer des « sortes » de fruitiers (formes sauvage et domestique, variétés différentes ou espèces proches). Cette différence de vocabulaire entre les deux auteurs reflète des préoccupations différentes et un projet différent. Dioscoride fait un inventaire des plantes utiles, tandis que Théophraste s'efforce de comprendre et de décrire l'organisation du monde végétal.

Données carpologiques, épigraphiques et littéraires ne couvrent pas également la période chronologique retenue pour ce travail. Seules les données archéobotaniques documentent toutes les périodes du Néolithique à l'époque romaine. Toutefois, elles sont moins abondantes pour le $1^{\text {er }}$ millénaire et font surtout défaut pour les époques classique, hellénistique et romaine. Au contraire, les sources écrites (littéraires et épigraphiques) se caractérisent par une certaines diversité et une relative abondance pour ces périodes, tandis qu'il n'existe aucune documentation écrite avant le Bronze récent, puis que toute la première moitié $\mathrm{du} 1^{\mathrm{er}}$ millénaire en est également dépourvue. A cela, il faut 
ajouter que les sites archéologiques comme les documents écrits sont de nature différente, et qu'il existe un lien entre chronologie et type de site, et entre chronologie et type de texte (traités de botanique, de médecine ou d'agronomie; inventaires de produits échangés et inventaires de cultures à entretenir sur un terrain). Si les textes nous renseignent sur la perception des fruits et des fruitiers, il est difficile de déterminer si les auteurs anciens ont réellement observés les éléments qu'ils décrivent. Au contraire, les restes archéologiques constituent la preuve tangible de la consommation de fruits, cependant ils ne permettent d'émettre aucune hypothèse sur leur perception.

\section{Résultats}

\section{Résultats des analyses carpologiques}

Le nombre de taxons par période est relativement constant : 13 taxons pour le Néolithique et le Bronze ancien, 12 pour le Bronze récent et pour l'âge du Fer, 11 pour la deuxième moitié du $1^{\text {er }}$ millénaire (figure $2 \mathrm{a}$ ). En revanche, la liste des taxons varie au cours du temps, bien que certains fruits soient attestés à toutes les périodes. La distribution par région est plus contrastée : les sites de Grèce du nord (Macédoine et Thrace, auxquelles s'ajoutent les îles du nord de l'Egée) ont livré 18 taxons fruitiers, ceux de Grèce centrale 13 et ceux du Péloponnèse 12. En revanche, les 13 sites de Thessalie n'ont livré que neuf taxons (figure $2 b$ ).

Ces différences s'expliquent d'abord par des nombres de sites différents d'une région à l'autre. Les régions où la richesse taxinomique est la plus importante sont celles où le nombre de sites investigués est le plus important. Ensuite, le nombre de périodes représentées dans les différentes régions est également un facteur qui peut expliquer ces différences de richesse taxinomique : alors que toutes les périodes sont représentées dans le nord de la Grèce, en Grèce centrale

Figure 2 : Nombre de taxons a) par période b) par région.

Figure 2: Number of taxa a) across the different periods $b$ ) across the different regions. et dans le Péloponnèse, le Néolithique est surreprésenté en Thessalie, en particulier le Néolithique ancien, tandis qu'un seul site du Bronze et deux de l'âge du Fer représentent les autres périodes dans cette région (figure 1).

Parmi les taxons fruitiers pris en compte dans l'analyse en présence/absence, la vigne (Vitis vinifera) et le figuier (Ficus carica) sont attestés à toutes les périodes et dans toutes les régions. Ces taxons sont les plus fréquemment attestés : les pépins de raisin et les akènes de figues apparaissent dans respectivement 71 et 54 phases d'occupation, et leur fréquence reste stable du Néolithique à l'époque classique (figure 3). Vigne et figuier sont également représentés dans toutes les régions, et le nombre d'occurrences de leurs restes varie de l'une à l'autre plus ou moins proportionnellement au nombre de phases d'occupation comptabilisé dans chaque région (figure 4). L'olivier (Olea europaea) et le chêne (Quercus sp.) sont également attestés à toutes les périodes et dans toutes les régions. 44 phases d'occupation ont livré des noyaux d'olives et ceux-ci deviennent plus fréquents à partir de l'âge du Bronze. Peu fréquents dans le Nord de la Grèce, ils font en revanche communément partie des assemblages de Grèce centrale, du Péloponnèse, de Crète et des Cyclades, avec une fréquence comparable à celle des pépins de vigne. Les glands de chêne sont fréquents au Néolithique, puis le deviennent davantage à partir de l'âge du Bronze. On en trouve surtout dans le Nord de la Grèce (MacédoineThrace : 12 phases d'occupation sur 29; Thessalie : 9 phases d'occupation sur 22).

D'autres fruits attestés au Néolithique semblent devenir plus rares à partir de l'âge du Bronze récent. C'est le cas tout d'abord des pistaches (Pistacia sp.) et de l'amande (Prunus dulcis). Les pistaches sont attestées principalement dans le nord de la Grèce (12 occurrences sur 19) et en Thessalie (6); les attestations d'amandes se répartissent entre la Crète (6), le Péloponnèse (5), la Macédoine (4), la Thessalie (3) (figure 4). Le fruit du cornouiller mâle

(b)

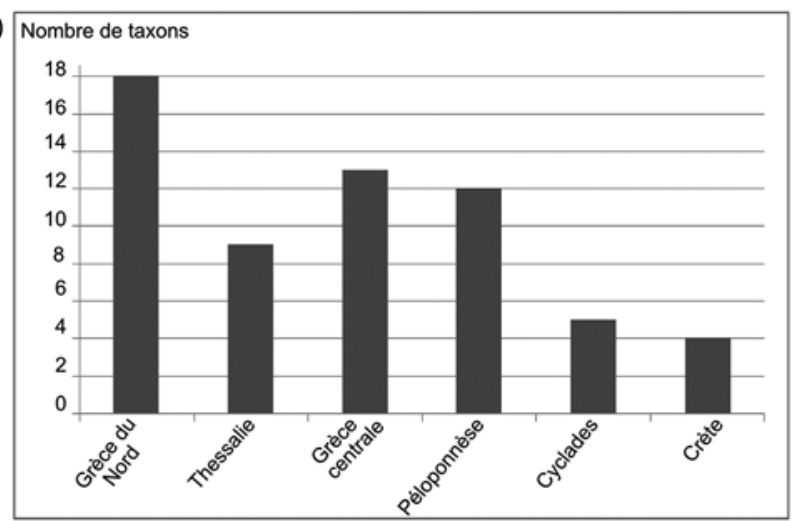




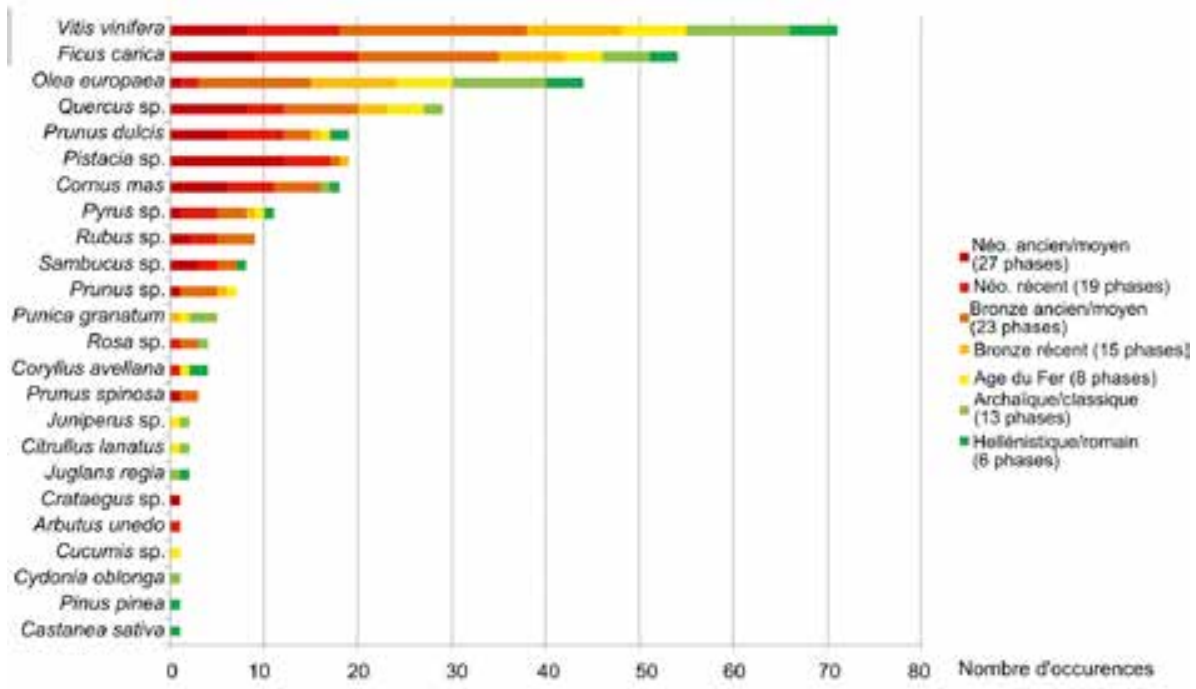

Figure 3 : (Voir planche couleur $\bullet \bullet$ ) Nombre d'occurrences de taxons fruitiers par période établi d'après le corpus carpologique (nombre total de phases chronologiques : 111).

Figure 3: (See colour plate $\bullet \bullet$ ) Number of records of fruit across the different periods based on seeds and fruit remains (total number of chronological phases: 111).

Figure 4: (Voir planche couleur $\bullet \bullet)$ Nombre d'occurrences de taxons fruitiers par région établi d'après le corpus carpologique (nombre total de phases chronologiques : 111).

Figure 4: (See colour plate $\bullet$ ) Number of records of fruit across the different regions based on seeds and fruit remains (total number of chronological phases: 111).

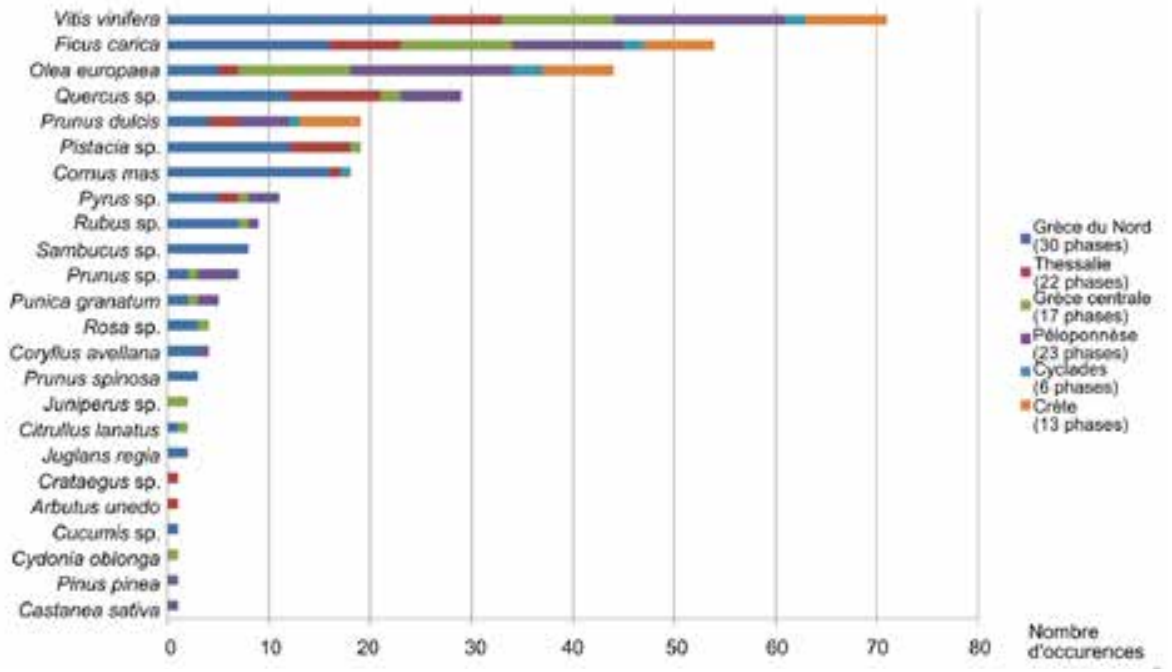

(Cornus mas), ensuite, semble caractéristique des périodes anciennes (Néolithique ancien - Bronze ancien) et du Nord de la Grèce : sur 18 phases d'occupation où il est attesté, 16 relèvent de sites de Macédoine. Les baies de sureau (Sambucus sp.) et les mûres de ronce (Rubus sp.) sont surtout présentes au Néolithique et au Bronze ancien et exclusivement dans le Nord de la Grèce (figures 3 et 4). La poire (Pyrus sp.) est également plus fréquente sur les sites du nord de la Grèce qu'ailleurs (5 attestations en Macédoine) et plus fréquente au Néolithique récent qu'aux périodes postérieures (respectivement quatre et trois occurrences pour le Néolithique récent et le Bronze ancien/moyen, contre une ou aucune aux autres périodes).

Enfin, la grenade (Punica granatum) est attestée sur cinq phases d'occupation. La plus ancienne remonte au Bronze récent : quelques graines ont été découvertes dans les niveaux du Bronze récent de Tirynthe (Kroll, 1982, 1984).

Les autres taxons (Prunus sp., Rosa sp., Corylus avellana, Citrullus lanatus, Juniperus sp., Arbutus unedo, Crataegus sp., Cucumis sp., Cydonia oblonga, Juglans regia, Castanea sativa et Pinus pinea), sont présents dans moins de cinq phases d'occupation, il est donc difficile de proposer des observations sur leur fréquence à l'échelle de la Grèce (figure 3 et 4). Plusieurs de ces fruits proviennent uniquement de contextes rituel ou funéraire. Les restes de noix (Juglans regia) proviennent de contexte funéraire (Thasos, $4^{\mathrm{e}}$ siècle avant notre ère, Megaloudi, 2006, 2016; Megaloudi et al., 2007), les seuls restes de châtaigne (Castanea sativa) et de pin pignon (Pinus pinea) d'un contexte rituel d'époque hellénistique à Messène dans le Péloponnèse (Megaloudi, 2005, 2006, 
2016), et enfin la seule graine de coing (Cydonia oblonga), dont l'identification n'est pas certaine (il pourrait s'agir d'un pépin de pomme ou de poire : il est en effet difficile de distinguer les pépins de ces trois espèces) vient des dépôts sacrificiels de Delphes, datés du viII ${ }^{\mathrm{e}}$ siècle (Luce et Marinval, 2008).

Les carporestes provenant de l'Heraion de Samos et datés du viI ${ }^{e}$ siècle avant notre ère (Kučan, 1995, 2000) attestent la présence de plusieurs fruits supplémentaires : la pêche (Prunus persica), le myrte (Myrtus communis), la mûre de mûrier noir (Morus nigra). Ces taxons sont donc attestés seulement sur l'île de Samos et uniquement pour l'époque archaïque, ce qui ne permet aucunement d'affirmer leur culture en Grèce dès le viII ${ }^{\mathrm{e}}$ siècle ni même leur consommation en dehors du contexte religieux.

\section{Épigraphie mycénienne}

Les seuls fruits mentionnés dans les tablettes sont les olives, désignées par l'idéogramme * 122 (42 mentions) et les figues, désignées par le syllabogramme ${ }^{*} N I$ (41 mentions). Olives et figues apparaissent dans les rations alimentaires distribuées aux travailleurs, sans pour autant en être des éléments récurrents contrairement aux céréales. Elles apparaissent également dans les listes d'offrandes religieuses ou dans les listes de denrées pour les banquets et les fêtes religieuses : les transactions contrôlées par le palais touchent davantage aux activités sacrées que domestiques (e.g. Bennett, 1958; Bendall, 2007), toutefois, ces produits n'ont certainement pas un usage uniquement rituel au Bronze récent. D’autre part, ces fruits sont impliqués dans d'autres activités que l'alimentation, manifestement soumises au contrôle de l'autorité palatiale, comme l'artisanat des textiles et la fabrication d'huile, de parfums et d'onguents.

Parmi les arbres potentiellement mentionnés, seuls sont bien identifiés le figuier et la vigne. Le premier est désigné par le terme su-za (grec classique sykê - бvкñ), présent dans cinq documents. Dans deux textes, on trouve le figuier associé à la vigne. Celle-ci est désignée par le terme we-je-we (hyiè - viń; KN Gv 863 et PY Er 880). On trouve également le terme wo-na-si (oinas - oivóc), "vignoble ", dans une tablette Knossos (Gv 863; Ventris et Chadwick, 1973; Killen et Olivier, 1989, 215; Palmer, 1994; Killen, 2004).

Il est possible que l'olivier soit également mentionné dans les tablettes, sous le terme $e$-ra-wa (elaia - $\dot{\varepsilon} \lambda \alpha i ́ \alpha)$ (Duhoux, 1993).

On rencontre également des mentions d'arbres malheureusement non identifiés. L'idéogramme * 174 pourrait désigner de jeunes arbres, dont l'espèce peut être précisée par un mot ou un idéogramme associé (Palmer 1994, 47-48); et l'idéogramme * 176 communément appelé ARB possède la particularité de présenter de légères différences d'une mention à l'autre. Selon le contenu du document où cet élément apparait, plusieurs interprétations ont été proposées (Melena, 1983, p. 102-103; Palmer, 1994, p. 46). Quoi que désignent ces deux idéogrammes, ils sont le signe d'une exploitation et d'un contrôle par le palais de fruitiers autres que la vigne et le figuier.

Enfin, l'huile d'olive et le vin tiennent une place relativement importante dans les listes de produits. C'est l'idéogramme ${ }^{*} 130$ qui désigne l'huile, ainsi que le mot e-ra-wo (élaion - Énatov). Un total de 131 documents contient l'idéogramme ou le mot. Le vin est désigné par l'idéogramme ${ }^{*} 131$ (56 tablettes et quatre nodules où il apparaît ont été relevés), ainsi que le terme wo-no (oinos - oî̀voc; PY Vn 20; Palmer 1994, p. 60-61). Bien qu'aucune mention de raisin ne soit connue, il est probable que cela faisait partie du régime alimentaire des habitants des régions contrôlées par les palais mycéniens.

\section{Baux ruraux}

La vigne, le figuier et l'olivier sont les fruitiers les plus régulièrement mentionnés et dénombrés dans les baux ruraux.

La vigne apparait dans 67 textes sur 84 sous le terme ámpe-

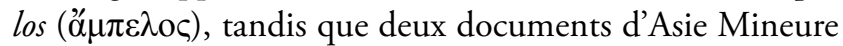
emploient le terme phuteia ampelinè ( $\varphi \tau \tau \varepsilon i ́ \alpha ~ \alpha ُ \mu \pi \varepsilon \lambda i ́ v \eta ~: E A$ 25 (1995), n 9, 1. 6; Sinuri, 47b, 1. 15), "plantation de vignes ".

43 textes mentionnent des figuiers. Le terme le plus

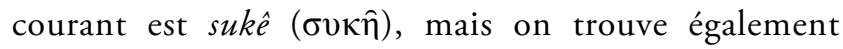
d'autres expressions dans les baux d'Asie Mineure : déndra

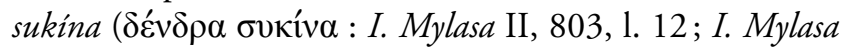


211, 1. 12-13).

Quant à l'olivier, plusieurs termes peuvent le désigner mais il est plus rarement mentionné dans les baux que la

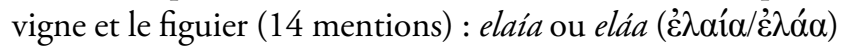
est le plus fréquent (Attique : $I G \mathrm{II}^{2}, 2492,2493$ et $I G^{3}$, 84, 1. 33; Délos : ID 366 B, ID 1416; Gazôros : Nouveau

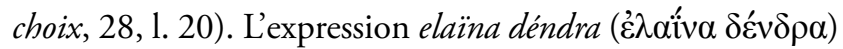
apparaît dans sept inscriptions d'Asie Mineure (I. Mylasa I 207A, 1. 8; I. Mylasa I, 207B, 1. 14; I. Mylasa I, 214, 1. 3; I. Mylasa II, 803, 1. 12, I. Mylasa II, 814, 1. 9, Sinuri, 47b, 1. 3; Sinuri, 56C, 1. 1).

On trouve deux autres expressions dans une inscription provenant de Délos et relatives à un domaine de Mykonos :

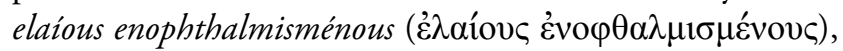
qui désigne des oliviers greffés, au nombre de 87 sur ce 
terrain, et elaious ('̇̉aíovৎ) seul, dont le nombre n'est pas certain (100 ?). Cette expression lacunaire a été interprétée comme une mention d'oliviers sauvages (Kent 1948; ID 366 B) : en effet, ce terme désigne un olivier différent de elaía ainsi que de elaíous enophthalmisménous. Il peut s'agir d'une autre variété, ou d'un arbre ayant un statut différent, ni cultivé, ni greffé. Or dans une autre inscription relative à un domaine de Mykonos, le terme agri[elaious]

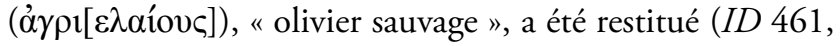
$\mathrm{Bb}, 1.55-57)$, et l'association des arbres greffés et sauvages paraît cohérente compte tenu de l'utilisation d'oliviers sauvages comme porte-greffe durant l'Antiquité (Brun 2003, p. 128; Théophraste, Causes des phénomènes végétaux $1,6,10$ ).

Le terme erinós - દ́pıvóc apparaît dans les inscriptions de Délos. Ce terme désigne le caprifiguier, ou « figuier mâle »: celui-ci est utilisé comme pollinisateur pour féconder les figuiers femelles. Dans neuf inscriptions sur dix, le terme erinós est associé au figuier (IG XI 2, 287A, 1. 155, 157, 169 et 174 ; ID 374 Aa, 1. 27; ID 374 Ab, 1. 1; ID 452, l. 20 et $24 ; I D 1416 \mathrm{~B}, 1.45)$. Ce pourrait être un indice en faveur de l'utilisation de variétés non parthénocarpiques, qui ont donc besoin de l'apport en pollen d'un caprifiguier, et de la pratique de la caprification. Le texte de Théophraste atteste d'ailleurs cette pratique pour l'Antiquité (Recherches sur les plantes 2, 8, 1-3).

Sept autres arbres peuvent être identifiés et sont mentionnés entre cinq et une seule fois. Le grenadier (rhoá ou rhoè - $\rho$ ód/poń) est mentionné cinq fois dans des inscriptions relatives à des baux de Délos et Rhénée (: $I G \mathrm{XI}, 2$, 287A, 1. 147 et 155 ; ID 373 B, 1. 40 ; ID 374 Aa, 1. 20 et $\mathrm{Ab}, 1.1$ ). On compte trois mentions de myrte (mursinè

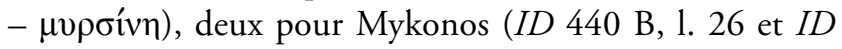
$461 \mathrm{Bb}, 1.55-57$ ) et une pour Thasos (IG II Suppl., 353,

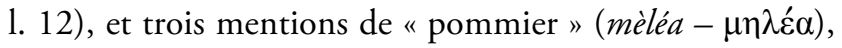
qui concernent toutes des domaines de Mykonos (ID 366 B, 1. 23; ID 440 B, 1. 50; ID 461 Bb, 1. 55-57). Aucun pommier sauvage n'est endémique des Cyclades, toutefois le pommier domestique (Malus domestica) a pu être introduit et mis en culture. Le terme mèléa est problématique et la lecture des textes littéraires permettra de préciser la nature de ce problème plus loin.

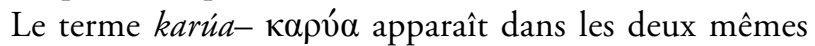
inscriptions relatives à Mykonos, tandis que M. Launey a restitué le terme leptokaruá - $\lambda \varepsilon \pi \tau$ - $\alpha \rho \rho \alpha ́$ dans le bail du jardin d'Heraklès à Thasos (IG II Suppl., 353, 1. 12; Launey, 1937). Ces termes désignent vraisemblablement un arbre à fruit à coque, noyer, noisetier ou amandier (Athénée, Deipnosophistes 2, 52a-b; Th. Rech. 3, 15, 1 : Pline, Histoire Naturelle 15, 86-94).
Enfin, sont recensées une mention de palmier (phoînix deux à Mykonos (ID 452, 1. 29), ainsi qu'une mention de

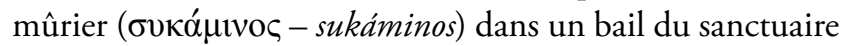
de Sinuri en Asie Mineure (Sinuri, 47a, 13). L'absence de précision quant à ce palmier ne permet pas de déterminer de quelle espèce il s'agit. Il existe un palmier endémique de Crète, Phoenix theophrasti, qui peut pousser dans les îles de la mer Egée. Mais il est possible que des palmiers-dattiers (Phoenix dactylifera) aient été introduits dans les régions les plus méridionales de Grèce où le climat leur permet de fructifier (Amigues 2003a, p 133, n. 19). Toutefois il pourrait s'agir d'un arbre à palme d'un genre autre que Phoenix, comme on le verra dans l'analyse des noms d'arbres dans les textes littéraires.

Le nombre d'arbres présents ou à planter est parfois mentionné. Il varie selon les baux et selon les espèces. Ainsi, les pieds de vigne se comptent par centaines voire par milliers dans les domaines d'Apollon à Délos, Rhénée et Mykonos. Les figuiers ne dépassent 100 individus qu'à Mykonos où 143 arbres sont comptés dans le domaine de Thaleon (ID 366, B, 1. 17-18). Dans le même document, 147 oliviers, 100 " oliviers sauvages " (elaious) et 87 " oliviers greffés " sont dénombrés. Une inscription d'Athènes précise le nombre d'oliviers que le locataire doit planter (100:IG $\mathrm{I}^{3}$ 84, 1. 33). Quatre baux de Mylasa, manifestement tous relatifs au même bien-fonds (Pernin, 2014, 316), signalent trois oliviers (I. Mylasa I 207A, 207B, 210 et 214). Ailleurs, les oliviers ne sont pas dénombrés, et le nombre de figuiers varie entre quatre arbres (IG XI 2, 287A, 1. 147) et 73 (ID 351, 1. 10-11).

Les figuiers sauvages ou caprifiguiers sont nettement moins nombreux : entre un et cinq (Délos, $I G$ XI 2, 287 A, 1. 151-152 [Brunet, 1990]). Quant aux autres arbres, le grenadier est toujours seul; le myrte est planté par deux, sauf à Thasos où il est prescrit d'en planter dix pieds, soit autant que de figuiers. D'après les baux relatifs à Mykonos, les " pommiers " peuvent aller jusqu'à 101 (selon J.H. Kent : 1948, ID 366, B, 1. 23 - nombre jugé douteux : Bruneau et Fraisse, 1981). Les noyers/noisetiers/amandiers sont attestés par deux, le palmier et le mûrier seuls.

Outre les arbres nommés, on trouve un certain nombre de mentions d'arbres indéterminés. Ces derniers ne sont

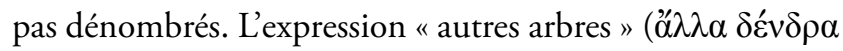
- álla déndra) apparaît 14 fois, dans des textes qui mentionnent par ailleurs des arbres déterminés - souvent vigne ou figuier : à Rhamnonte ( $\left.I G \mathrm{II}^{2}, 2494\right)$, sur sept baux de Mylasa (I. Mylasa I, 207A et B, 210, 211, 214; Sinuri, 51, 56) et sur six baux d'Olymos (I. Mylasa II, 803, 805, 806, 
810, 814; EA 25 (1995), 9, p. 47). Il s'agit d'une catégorie large qui englobe tous les arbres sauf ceux déjà cités.

Un ensemble de 13 textes mentionne des " arbres " (dén$d r a-\delta \varepsilon ́ v \delta \rho \alpha)$ sans autre précision. Il s'agit de textes qui restent très généraux, dans lesquels il est écrit que le terrain comprend des arbres, qu'il faudra entretenir et présenter à la fin du bail, sans en préciser l'espèce (Athènes, $I G \mathrm{II}^{2}, 2499$, 1. 15; Aixoné, $I G \mathrm{II}^{2}$, 2492, 1. 16-17; Thespies, I. Thesp 54, 1. 4; Mylasa, I. Mylasa I, 216). Dans plusieurs baux d'Asie mineure, déndra semble désigner tous ce qui n'est pas de la vigne (I. Mylasa I, 204, 217; I. Mylasa II, 822, Sinuri, $47 \mathrm{~b}, 53,56)$. Cela peut servir à apporter une précision sur les arbres du terrain qui ont déjà été mentionnés auparavant sans énumérer de nouveau la liste des espèces, comme c'est le cas sur une inscription de Mylasa (I. Mylasa I, 206). Toutefois, il est possible que dans cet inventaire de mentions du terme " arbres » sans précision, certaines aient été accompagnées de l'adjectif álla, non conservé.

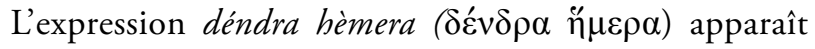
dans trois inscriptions. L'adjectif hèmeros (ท̆ $\mu \varepsilon \rho \circ \varsigma)$ signifie " cultivé ", par opposition à ágrios (ärpıc), " sauvage ". On peut supposer qu'il s'agit d'arbres fruitiers autres que la vigne, qui est généralement nommée (Myrrhinonte, $I G$ $\mathrm{II}^{2}, 1241$, 1. 23; Rhamnonte, $I G \mathrm{II}^{2}$, 2493, 1. 18, Delphes lotissement, 1. 12).

Une autre « catégorie » d'arbres fruitiers apparât dans un

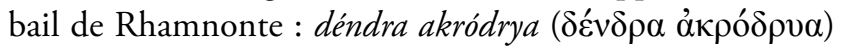
$\left(I G \mathrm{II}^{2}, 2494,1.12\right)$. Le texte stipule que ces arbres doivent être entretenus.

Enfin, le bail de Gazôros (Nouveau choix, 28, 1. 10-13) mentionne deux autres catégories : déndra karpofóra $(\delta \varepsilon \dot{v} \delta \rho \alpha$

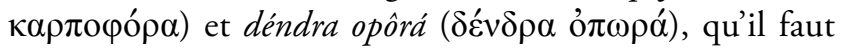
planter, tout comme la vigne. Le texte définit la manière dont le cultivateur et la cité se partagent la récolte : pour la vigne, chacun en récupère la moitié, pour les oliviers, le cultivateur obtient les deux tiers, pour les figuiers et les opôrá, celui qui s'en occupe bénéficie de l'intégralité de la récolte. Les déndra opôrá sont donc mis sur le même plan économique que les figuiers. Les déndra karpofóra semblent quant à eux être distincts de la vigne et des opôrá. Littéralement, cela signifie "arbre qui porte des fruits" : supposant sans doute que c'est une manière d'insister sur la production de ces arbres, C. Vatin traduit ce terme par " arbres de rapport " (Vatin, 1962). Il suppose que cela désigne peut-être les oliviers, qui ne sont pas mentionnés dans la liste d'essence à planter, mais apparaissent dans le partage de la récolte (où les déndra karpofóra n'apparaissent pas alors qu'ils apparaissaient dans la liste d'arbres à planter).

\section{Textes littéraires}

La lecture des textes de Théophraste et Dioscoride permet d'établir une liste d'une quarantaine de taxons fruitiers exploités pour la médecine ou l'alimentation, dont il n'est pas certain que tous soient cultivés en Grèce entre le $\mathrm{IV}^{\mathrm{e}}$ siècle avant notre ère et le $\mathrm{II}^{\mathrm{e}}$ siècle de notre ère. En effet, Théophraste fait référence à des plantes asiatiques et Dioscoride cherche à énumérer les vertus et propriétés du plus grand nombre de plantes possible, y compris celles qui ne poussent pas en Grèce. Les descriptions de Théophraste sont souvent assez précises pour identifier les plantes décrites à l'espèce.

On peut distinguer les arbres courants, bien connus des anciens et manifestement mis en culture, ceux qui ne semblent pas cultivés et ceux pour lesquels l'usage dans l'alimentation est incertain.

Les arbres les plus fréquemment cités dans les livres consacrés aux arbres cultivés des Recherches sur les plantes de

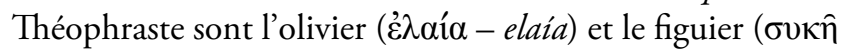
- sukê). Ces arbres étaient alors, d'après les sources littéraires d'époque classique mais également d'après les textes homériques, très courants (Amigues, 2016). Très souvent associé à

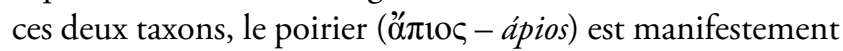
très répandu également. Pour ces trois arbres, Théophraste distingue des formes sauvages et des formes domestiques, de même que Dioscoride.

La vigne est mentionnée par Théophraste qui laisse deviner qu'elle était courante également, de même que le grena-

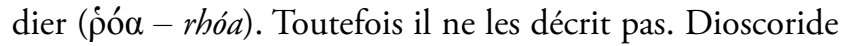
mentionne deux sortes de grenades : la douce et l'acide ( $\dot{\eta}$

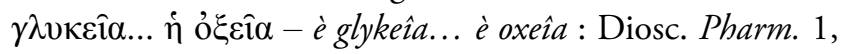
$110,1)$ et distingue la vigne sauvage de la vigne cultivée (Diosc. Pharm. 5, 2).

Parmi les arbres cultivés, Théophraste mentionne encore le pommier (mèléa - $\mu \eta \lambda \dot{\varepsilon} \alpha$ : Th. Rech. 2, 1, 2; 2, 2, 5), l'amandier $(\dot{\alpha} \mu v \gamma \delta \alpha \lambda \hat{\eta}$ - amygdalê : Th. Rech. 2, 2, 5) et le

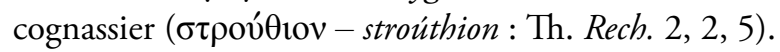

Vigne, figuier, olivier, grenadier, poirier et pommiers sont déjà mentionnés dans l'Odyssée. Dans trois passages, ces arbres sont associés (Odyssée 7, 112-116 et 122-126; 24, 245-247; 24, 340-342). Tous sont désignés par les mêmes termes qu'en grec classique, excepté le poirier, qu'Homère nomme onchnè (ö $\gamma \chi \vee \eta)$. Dioscoride range le pommier, l'amandier et le cognassier dans la même catégorie, celle des "pommiers» (Diosc. Pharm. 1, 115). On trouve dans cette même catégorie les " pommes douces" $(\mu \varepsilon \lambda i ́ \mu \eta \lambda \alpha-$ melímèla : Diosc. Pharm. 1, 115, 3 - aucune description ne peut nous aider à identifier ce dont il s'agit), les « pommes d'Arménie ", ('A $\rho \mu \varepsilon v 1 \alpha \kappa \alpha ́$ - Armeniaká : Diosc. Pharm. 1, 
$115,5)$ qui seraient peut-être des prunes, et les " pommes de Perse " (Diosc. Pharm. 1, 115, 4). Plus loin, il précise que les «pommes de Perse " peuvent également être appelées

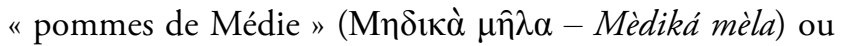
kedromèla (Diosc. Pharm. 1, 115, 5). Sa description ainsi que les textes de Théophraste, qui le range parmi les fruits exotiques (Th. Rech. 4, 4, 2), permettent d'identifier un agrume d'espèce indéterminée (Pagnoux et al., 2016; Pagnoux, 2017). Cet agrume est identifié comme un cédrat dans la littérature, à cause de la description qu'en fait Dioscoride : «[...] le fruit quant à lui est allongé, ridé, de couleur dorée et au parfum entêtant, avec des graines semblables à celles de la poire. " (Diosc. Pharm. 1, 115, 5). Plus tard, au III siècle de notre ère, Galien décrit un fruit en trois parties : «celle, acide, qui se trouve au centre, celle qui entoure cela, en quelque sorte la chair de ce fruit, et la troisième qui les recouvre, l'enveloppe externe qui entoure ce fruit " (Galien, Propriétés des aliments 2,37). On peut reconnaitre un cédrat dont l'albédo (partie blanche qui se trouve entre l'écorce et la chair) est relativement épais. Toutefois, ces descriptions ne permettent pas d'affirmer que seul le cédrat était connu durant l'Antiquité. En effet, compte tenu des anomalies reproductives qui affectent les différentes espèces du genre Citrus et des possibilités de croisement entre espèce, d'autres espèces pouvaient croître durant l'Antiquité (Pagnoux et al., 2016, Pagnoux, 2017).

Le cortège des fruits sauvages exploités pour l'alimentation et/ou la médecine est relativement varié, d'après les auteurs antiques. On entend ici "sauvage " par opposition à "cultivé », et non "génétiquement sauvage ".

D'autres sortes de " pommes » apparaissent dans les textes et désignent des fruits sauvages. La "pomme de printemps " ('̌apıvó - eariná en grec; mala verna en latin) est mentionnée par Pline (Hist. Nat. 23, 100), Théophraste (Rech. 2, 1, 3 ) et Dioscoride (Pharm. 1, 115, 4). D'après S. Amigues, il pourrait s'agir du fruit du faux abricotier (Prunus cocomilia), qui pousse dans les montagnes du nord de la Grèce et produit un fruit de couleur jaune à maturité (Amigues 2003a,117, n. 8). La " pomme d'Epire ", qui apparaît chez Dioscoride ('Н $\pi \varepsilon \iota \omega \tau \imath \kappa \grave{\alpha}$ - Epeirôtiká [mêla]; Pharm. 1, $115,4)$, aussi appelée " orbiculata " (o’ $\rho \beta 1 \kappa \lambda \hat{\alpha} \tau \alpha-$ orbiklâta, Pharm. 1, 115, 4) est un fruit sauvage indéterminé.

Théophraste mentionne trois fruitiers appartenant à une "catégorie » qu'il nomme $\mu \varepsilon \sigma \pi i ́ \lambda \eta$ (mespilè : Th. Rech. 3, 12, 5). Il semble sous-entendre que les fruits des trois sortes de mespilè sont comestibles (Th. Rech. 3, 12, 2). La description des fruits ne permet pas de déterminer précisément à quelles espèces il fait référence dans ce passage, toutefois on peut y reconnaître le genre Crataegus (Amigues, 2003b, 165-166, n. 14 et 15), qui regroupe les aubépines et le néflier, dont l'an- cien nom scientifique était Mespilus germanica. Diocoride

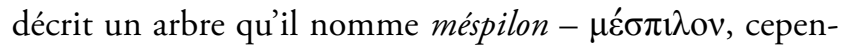
dant la description de son fruit ne permet pas d'identifier une nèfle avec certitude (Diosc. Pharm. 1, 118).

Théophraste évoque d'autres arbres, pourtant considérés comme sauvages dans sa classification, pour lesquels sont observées une forme domestique et une forme sauvage. Ce sont les fruits, manifestement consommés, qui permettent de les distinguer : ainsi les cornouilles mâles (kráneia) et les sorbes (ôua) sauvages sont meilleurs que les fruits des mêmes arbres lorsqu'ils sont cultivés (Th. Rech. 3, 2, 1). Quant au

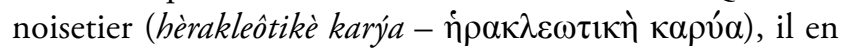
existe une forme domestique et une forme sauvage qui se distinguent par la qualité de leurs fruits et par la taille des feuilles (Th. Rech. 3, 15, 1).

Dioscoride évoque la consommation des fruits de deux autres fruitiers dont il est difficile de déterminer s'ils étaient cultivés en Grèce à l'époque romaine, ou si les fruits étaient importés. L'un pourrait correspondre au prunier domes-

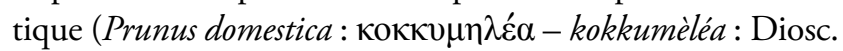
Pharm. 1, 121). Il précise notamment que les Syriens en consomment le fruit séché. Chez Théophraste, on ne trouve pas de mention de la consommation des fruits ni de la culture de l'arbre, bien qu'il le mentionne pour l'opposer à un prunier sauvage (Th. Rech. 3, 6, 4). Le deuxième est le "noyer royal ", désigné par ses fruits (kárua basiliká -

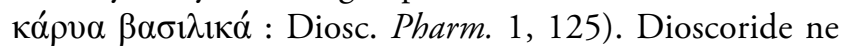
les décrit que brièvement et le terme est absent du texte de Théophraste.

Viennent ensuite plusieurs fruitiers pour lesquels Théophraste ne mentionne pas d'utilisation des fruits, mais que Dioscoride évoque dans ses recettes médici-

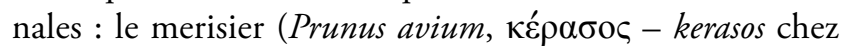

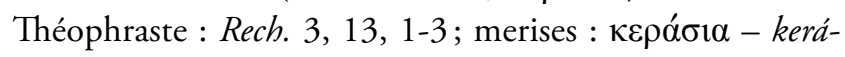
sia chez Diosoride, Pharm, 1, 113). Il n'est fait aucune mention ni chez l'un ni chez l'autre de cerisier cultivé. On trouve en revanche chez Athénée une citation de Diphile de Siphne, une source contemporaine de Lysimaque (successeur d'Alexandre le Grand), attestant la consommation de cerises, notamment de cerises de Milet, à la fin $\mathrm{du}_{\mathrm{IV}}{ }^{\mathrm{e}}$ ou au début du III ${ }^{\mathrm{e}}$ siècle avant notre ère (Ath. Deipn. 2, 51a-b).

De la même manière, Théophraste mentionne plusieurs sortes de ronces, mais ne signale pas que leurs fruits sont comestibles. Le terme $\chi \alpha \mu \alpha i ́ \beta \alpha \tau o c$ (chamaíbatos) désigne la ronce - il précise qu'il en existe plusieurs espèces, difficile à identifier (Th. Rech. 2, 18, 4). Il les rapproche de l'églantier (Rosa sp. : Th. Rech. 3, 18, 4), également mentionné par Dioscoride qui en distingue plusieurs sortes (Diosc, Pharm $1,94 ; 1,99 ; 4,38)$. 


\begin{tabular}{|c|c|c|c|}
\hline Carpologie & Epigraphie mycénienne & Epigraphie classique & Textes littéraires (Théophraste et Dioscoride) \\
\hline Amandier (Prunus dulcis) & Figuier (Ficus carica) & Figuier (Ficus carica) & Amandier (Prunus dulcis) \\
\hline Arbousier (Arbutus unedo) & Olivier (Olea europaea) & Grenadier (Punica granatum) & Arbousier (Arbutus unedo) \\
\hline Aubépine (Crataegus sp.) & \multirow[t]{27}{*}{ Vigne (Vitis vinifera) } & Laurier (Laurus nobilis) & Aubépine/néflier (Crataegus sp.) \\
\hline Châtaignier (Castanea sativa) & & Mûrier noir (Morus nigra) & Cade (Juniperus oxycedrus) \\
\hline Chêne (Quercus sp.) & & Myrte (Myrtus communis) & Chêne (Quercus sp.) \\
\hline Cognassier (Cydonia oblonga) & & Noisetier (Corylus avellana?) & Cognassier (Cydonia oblonga) \\
\hline Cornouiller mâle (Cornus mas) & & Noyer (Juglans regia?) & Cornouiller mâle? (Cornus mas?) \\
\hline Eglantier (Rosa sp.) & & Olivier (Olea europaea) & Eglantier (Rosa sp.) \\
\hline Figuier (Ficus carica) & & Palmier (Phoenix sp.?) & Figuier (Ficus carica) \\
\hline Genévrier (Juniperus sp.) & & Pommier (Malus sp.?) & Grenadier (Punica granatum) \\
\hline Grenadier (Punica granatum) & & Vigne (Vitis vinifera) & Merisier/cerisier (Prunus avium/cerasus) \\
\hline Melon/Concombre (Cucumis sp.) & & dendra hèmera & Noisetier (Corylus avellana) \\
\hline Mûrier noir (Morus nigra) & & (dendra) akrodrya & Noyer (Juglans regia) \\
\hline Myrte (Myrtus communis) & & dendra karpofora & Olivier (Olea europaea) \\
\hline Noisetier (Corylus avellana) & & dendra opôra & Palmier (Phoenix sp.? Autre genre?) \\
\hline Noyer (Juglans regia) & & & Pistachier lentisque (Pistacia lentiscus) \\
\hline Olivier (Olea europaea) & & & Pistachier térébinthe (Pistacia terebinthus) \\
\hline Pastèque (Citrullus lanatus) & & & Poirier (Pyrus sp.) \\
\hline Pêcher (Prunus persica) & & & Pommier (Malus sp.) \\
\hline Pin pignon (Pinus pinea) & & & $\begin{array}{l}\text { "Pomme d'Arménie " (Prunus armeniaca? } \\
\text { P. domestica?) }\end{array}$ \\
\hline Pistachier (Pistacia sp.) & & & $\begin{array}{l}\text { "Pomme de Médie " = " pomme de Perse " } \\
\text { (Citrus sp.) }\end{array}$ \\
\hline Poirier (Pyrus sp.) & & & «Pomme de printemps» (Prunus cocomilia?) \\
\hline Prunellier (Prunus spinosa) & & & «Pomme d'Epire» \\
\hline Prunier (Prunus sp.) & & & "Pomme douce » \\
\hline Ronce (Rubus sp.) & & & Prunier (Prunus domestica?) \\
\hline Sureau (Sambucus sp.) & & & Ronce (Rubus sp.) \\
\hline \multirow[t]{3}{*}{ Vigne (Vitis vinifera) } & & & Sorbier (Sorbus domestica? Sorbus sp. ?) \\
\hline & & & Sureau (Sambucus nigra? Sambucus sp.?) \\
\hline & & & Vigne (Vitis vinifera) \\
\hline
\end{tabular}

Tableau 2 : Listes des taxons attestés par les études carpologiques et les sources écrites.

Table 2: List of taxa attested by archaebotanical analyses and written sources.

Le pistachier térébinthe (Pistacia terebinthus) est mentionné sous le nom $\tau \varepsilon \rho \mu$ ív $\theta$ oc (terminthos) et décrit avec beaucoup de précision par Théophraste $(3,15,1)$ mais celuici ne dit rien de l'utilisation de ses fruits ni de son bois. Dioscoride énumère en revanche de nombreuses propriétés pour cet arbre (Diosc. Pharm. 1, 70). Ce dernier traite également du lentisque (Pistacia lentiscus, qu'il nomme $\sigma \chi i v o \varsigma$ - schinos: Pharm. 1, 69), mais beaucoup plus rapidement.

Le chêne constitue un cas un peu à part : ni Théophraste ni Dioscoride ne mentionnent explicitement la consomma- tion de ses fruits, cependant le premier écrit que le goût des glands est un critère permettant de distinguer plusieurs espèces (Th. Rech. 3, 8, 2-7).

Enfin, le cas du palmier est particulier. Théophraste lui consacre tout un chapitre du deuxième livre des Recherches sur les plantes (Th. Rech. 2, 6) car c'est un arbre singulier dont il liste les particularités. Ce chapitre montre que sous le terme phoinnix, Théophraste désigne des arbres à palmes qui n'appartiennent pas tous au genre Phoenix (Amigues, 2003b, 132-134). Phoînix désigne une catégorie d'arbres à palme 
dont certaines « sortes ", qui sont en fait des espèces appartenant à des genres différents, peuvent pousser en Grèce : le palmier nain (Chamaerops humilis), le palmier de Crète (Phoenix theophrasti) et peut-être le palmier-dattier (Phoenix dactylifera). Selon Dioscoride, le palmier pousse en Egypte. Il décrit succinctement son fruit qu'il désigne par le terme

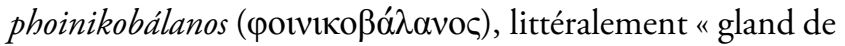
palmier» (Diosc Pharm. 1, 109).

\section{DisCussion}

Compte tenu du fait que l'ensemble des sources exploitées ne documente pas l'intégralité de la période étudiée, et que toutes les sources documentent des aspects différents de la culture des arbres fruitiers et de la consommation des fruits, leur mise en relation ne permet pas de proposer une évolution chronologique de la diversité fruitière en Grèce. Toutefois, précisément à cause de ce caractère discontinu des données, seule leur confrontation permet de mieux connaître les fruitiers exploités en Grèce ancienne. Le principal constat auquel nous conduisent les données mises en regard est le suivant : chaque type de donnée (carporestes, textes épigraphiques, textes littéraires) donne une image différente de la diversité fruitière. Les sources utilisées ne révèlent ni le même nombre de taxons ni les mêmes taxons (tableau 2).

On trouve la plus longue liste de taxons dans les textes littéraires : la littérature grecque d'époque classique et impériale permet d'attester, sinon la présence, tout au moins la connaissance, à ces périodes, de 29 fruitiers. Tous ne peuvent être identifiés à une espèce ou un taxon actuel connu. La carpologie atteste la présence, pour toutes périodes et toutes régions confondues, de 27 taxons fruitiers. L'épigraphie classique mentionne 11 arbres fruitiers et quatre catégories de fruitiers, et l'épigraphie mycénienne n'en mentionne que trois.

Ces différences d'un type de données à l'autre peuvent en partie être expliquées par la nature des enregistrements. La conservation et la découverte des restes de fruits résultent d'une succession de choix et de hasards : hasard de la disponibilité d'une plante dans l'environnement ou choix au moment de la cueillette, conditions aléatoires permettant la conservation, hasard de découverte liée au choix de la zone de fouille... La découverte de documents en linéaire $\mathrm{B}$ résulte du hasard lié aux fouilles, mais tient également à l'incendie qui a favorisé la conservation des tablettes. Ces " hasards » font suite à des choix d'inscription : les fruitiers et fruits concernés sont ceux dont la production intéresse l'autorité palatiale sur le plan de son fonctionnement économique et commercial. Les fruitiers mentionnés dans les baux ruraux sont également le résultat de plusieurs choix : seuls les contrats relatifs aux terrains publics ou sacrés sont gravés, et seuls les fruitiers plantés sur ces domaines et qui constituent un apport à la valeur du terrain (vente de leurs fruits, obtention d'arbres vigoureux et productifs grâce aux soins prodigués par le locataire) sont connus. Enfin, les plantes dont les mentions et les descriptions nous sont parvenues dans des traités de botanique ou de médecine sont le résultat de choix de la part des auteurs (plantes particulières, difficiles à identifier, faisant l'objet d'une culture spécifique, présentant des propriétés particulièrement intéressantes...). Naturellement, la conservation et la transmission au cours des siècles de ces écrits est également le résultat de choix et de hasards dont les causes nous échappent en grande part.

Quoi qu'il en soit, la nature des enregistrements conditionne non seulement le type, mais aussi le nombre de fruitiers mentionnés. Il n'est pas surprenant, en effet, que la carpologie et la littérature botanique et médicale portent à notre connaissance davantage d'espèces que les listes de produits échangés au sein des sociétés palatiales ou les inventaires de terrains à louer. En effet, malgré les biais qui ont été évoqués, les carporestes reflètent la consommation par les sociétés humaines des fruits comestibles à leur disposition, selon des choix dont les motivations nous sont inconnues mais conduisent, d'après les résultats des études carpologiques, à une large exploitation des ressources disponibles. De même, la description des arbres fruitiers a pour seule limite l'accès de leur auteur à son sujet et l'intérêt que celui-ci présente à ses yeux.

On peut souligner le fait que trois taxons seulement sont attestés par toutes les sources: la vigne (Vitis vinifera), le figuier (Ficus carica) et l'olivier (Olea europaea). Ces trois fruitiers se distinguent en effet des autres : ce sont les plus fréquents, et la vigne et le figuier sont généralement les plus abondants.

\section{Vigne, figuier, olivier}

Sur la base de plusieurs arguments, on peut supposer que la vigne et le figuier sont mis en culture dès le Néolithique. Ces indices sont l'abondance de restes sur plusieurs sites, l'association avec des charbons de bois et la récurrence des découvertes de ces espèces pour une même période. Ils ont davantage de poids s'ils sont associés (Ruas, 1996; Ruas et al., 2005). Dès le Néolithique ancien, on enregistre la découverte associée de pépins de raisin et d'akènes de figues avec des charbons de vigne et de figuier (notamment à Achilleion, Thessalie : Renfrew, 1989). La découverte de charbons de bois est en effet un indice de l'utilisation de bois issu de la taille de ces fruitiers, et peut donc refléter leur entretien (Miller, 2008). Au Néolithique récent, les découvertes de grandes quantités de restes se multiplient, notamment à 
Makri, en Thrace (Ntinou et Badal, 1998; Ntinou, 2002; Valamoti, 1998), et à Dikili Tash, en Macédoine, où plus de 2000 pépins ont été découverts associés à des restes de pressage (Valamoti et al., 2007, Valamoti, 2015; Valamoti et al., 2015) et à des résidus de vin (Garnier et Valamoti, 2016).

A partir de l'âge du Bronze (fin du $3^{\mathrm{e}}$ et $2^{\mathrm{e}}$ millénaires avant notre ère), le nombre de sites où vigne et figuier sont découverts en abondance augmente, ainsi que les indices en faveur du stockage et de la transformation de ces fruits (Toumba Thessalonikis : Andreou et Kotsakis, 1997; Mangafa et al., 1998 et Kastanas : Kroll, 1979, 1983; Macédoine; Lerne dans le Péloponnèse : Hopf, 1961 et 1962; Monastiraki en Crète : Fiorentino et Solinas, 2006; Sarpaki, 2012). Il est donc possible de supposer que ces fruitiers sont alors exploités de manière intensive, voire mis en culture dans toute la Grèce. Cette exploitation a pu consister, dans un premier temps, en l'entretien des arbres dans leur milieu naturel, ou en la transplantation de pieds sauvages dans l'espace cultivé.

Enfin, à partir du Bronze récent (1400-1200), la culture de la vigne et du figuier est attestée par trois tablettes en linéaire $\mathrm{B}$ trouvées à Knossos, qui évoquent des plantations d'arbres, parmi lesquels ces deux espèces, ainsi que d'autres arbres dont l'identification reste problématique comme on l'a vu plus haut.

L'olivier est d'abord attesté dans le sud de la Grèce et en Crète. La palynologie fait remonter sa présence en Crète à 5500-5000 avant notre ère (Moody et al., 1996; Bottema et Sarpaki, 2003; Pavlopoulos et al., 2005). Dans le sud du Péloponnèse, la présence de l'olivier est attestée à partir de 7000 avant notre ère (Kraft et al., 1980). Il est possible de faire remonter son exploitation en Crète au Néolithique récent d'après l'augmentation de la part de pollen d'olivier qui traduit l'expansion de la distribution de l'arbre. Sa culture débuterait réellement au Bronze ancien : la forêt semble reculer au profit d'une culture de céréales et d'oliviers, comme le laissent penser la diminution de la quantité de pollen de chêne à cette période et l'augmentation de ceux de graminées et d'oliviers. La découverte de charbons de bois dans plusieurs sites du l'âge du Bronze ancien plaide également en la faveur d'une exploitation de l'olivier à partir de cette période en Crète (ex. Myrtos, Renfrew, 1972), mais aussi dans les Cyclades (Akrotiri, à Théra : Sarpaki et Asouti, 2008; Dhaskalio sur l'île Kéros : Ntinou, 2013; Margaritis, 2013b - ces sites ont également livré des noyaux d'olives, dont la présence permet d'affirmer que les fruits étaient consommés). Dans le Péloponnèse, les premières attestions de restes carpologiques remontent au Bronze ancien, à Tirynthe (Kroll, 1982). Sa culture est supposée au Bronze récent dans le Péloponnèse et en Crète, d'après les tablettes de Knossos : bien que celles-ci ne proviennent pas du Péloponnèse mais de Crète, il est probable que la culture de l'olivier ait été pratiquée dans l'ensemble du monde mycénien.

Enfin, les charbons et les restes carpologiques laissent supposer une introduction de l'olivier dans le nord de la Grèce au $1^{\text {er }}$ millénaire lors de la colonisation de cette région par des Grecs venus de Corinthe et de l'île d'Eubée (Valamoti et al., 2018).

La production et la consommation de vin et d'huile d'olive a certainement joué un rôle dans le développement de la viticulture et de l'oléiculture. La découverte d'installations vraisemblablement utilisées pour la fabrication de vin et d'huile en Crète datées du Bronze moyen pour les plus anciennes, ainsi que les sources écrites mycéniennes (Bronze récent), ont été considérées comme des indices en faveur d'un lien entre arboriculture, produits transformés et développement de sociétés hiérarchisées (Renfrew, 1972; Gilman, 1981), d'abord en Crète, dès le Bronze moyen, puis dans le Péloponnèse et jusqu'en Thessalie au Bronze récent. Huile et vin auraient constitué des produits sur lesquels reposait la richesse d'une classe dirigeante, grâce aux possibilités d'échanges qu'ils représentaient. Une hypothèse plus nuancée consiste à supposer que les installations de transformation et leur présence dans des sites de type palais et villa à partir du Bronze moyen témoignent d'un changement dans les pratiques sociales et économiques de cette période : les besoins en huile et en vin, liés à une culture, des pratiques rituelles et des échanges commerciaux nouveaux, augmentent, et la fabrication et la gestion de ces produits sont organisées et contrôlées par une autorité politique centrale (Blitzer, 1993; Hamilakis, 1996). Avec l'expansion de la culture mycénienne hors du Péloponnèse et de Crète, l'usage de l'huile et le développement de l'oléiculture se propagent vraisemblablement.

A l'époque classique, l'olivier, la vigne et le figuier semblent garder la même place qu'au Néolithique et à l'âge du Bronze. Les sources épigraphiques permettent d'affirmer que ces trois fruitiers sont cultivés dans les Cyclades, sur l'île de Thasos, en Attique et en Asie mineure. Quant aux écrits de Théophraste, ils ne laissent aucun doute sur le fait que ces arbres sont cultivés et bien connus en Grèce classique. Vigne et olivier sont en outre présents avec la même fréquence dans les assemblages carpologiques d'époque classique et hellénistique qu'aux époques antérieures. D’après les baux, vigne et figuier apparaissent comme des éléments constitutifs des terrains à louer, qui apportent de la valeur au terrain et dont l'entretien revêt la même importance que celui des bâtiments.

La documentation épigraphique, l'état de la documentation carpologique et la hiérarchie établie au sein des ouvrages de Théophraste et Dioscoride font apparaitre ces trois frui- 
tiers comme les cultures dominantes. Les autres arbres semblent occuper une place secondaire. Cette hiérarchie peut s'expliquer par l'intérêt économique que présentent ces deux groupes : figuier, vigne et olivier produisent des aliments de base (les figues), des produits à valeur commerciale et rituelle (vin et huile); les autres produisent des fruits de garde, du bois et leurs productions peuvent sembler équivalentes les unes aux autres. Par ailleurs, on peut supposer, peut-être à compter du Bronze récent et avec certitude à partir de l'époque classique, le développement de "vignobles" où les pieds de vignes sont parfois très nombreux, jusqu'à plusieurs centaines, et plantés en rangs. Les tablettes mycéniennes mentionnent de nombreux pieds de vignes, de même que les inscriptions classiques. Plusieurs baux d'Asie Mineure, datés de l'époque hellénistique ( $\mathrm{II}^{\mathrm{e}}$ siècle avant notre ère), mentionnent également des rangs de vignes. Sur le site de Komboloi (Macédoine) des fosses parallèles, correspondant peut-être à des fosses de plantation, ont été mises au jour et interprétées comme l'empreinte d'un vignoble (Poulaki, 2003; Boissinot, 2013; Margaritis, 2015, 2016). Cette hypothèse repose également sur la découverte dans le même site de nombreux restes de vigne et résidus de pressage (Margaritis et Jones, 2006; Margaritis, 2015, 2016).

\section{Fruits du Néolithique}

Un deuxième ensemble de fruitiers pourrait avoir été mis en culture à partir du Néolithique, mais les indices de leur exploitation au-delà de l'âge du Bronze sont encore plus minces que ceux qui documentent la vigne, l'olivier et le figuier.

D'après les analyses carpologiques, un certain nombre de taxons semblent particuliers au Néolithique et au nord de la Grèce. Ces fruitiers semblent devenir plus rares après l'âge du Bronze, voire de disparaître des assemblages carpologiques aux périodes historiques. Cette situation est peutêtre due à l'état de la recherche, ainsi il convient de traiter les résultats obtenus dans ce travail avec prudence. Toutefois, on peut proposer quelques hypothèses sur leur culture.

Un premier ensemble est formé par le cornouiller mâle (Cornus mas), le sureau (Sambucus sp.), la ronce (Rubus sp.), et le poirier (Pyrus sp.). D'après les données carpologiques, ces fruitiers sont presque tous associés aux sites néolithiques du Nord de la Grèce. La lecture des textes grecs de Théophraste et Dioscoride permet de constater que leurs fruits sont toujours consommés 3000 ans plus tard, mais aucun de ces fruitiers n'est mentionné parmi les éléments à entretenir selon les clauses de location des terres publiques et sacrées. L'absence de certains de ces taxons dans les baux ruraux d'Attique et des Cyclades s'explique par leur grande rareté voire leur absence dans ces régions : c'est le cas notam- ment du cornouiller mâle (Cornus mas) qui n'y trouve pas les conditions écologiques pour se développer.

On peut ajouter à cette liste le chêne (Quercus sp.) et le pistachier (Pistacia sp.), qui font partie, au Néolithique, des fruitiers les plus fréquemment attestés dans les assemblages carpologiques. Le chêne se raréfie après le Bronze moyen. Théophraste suggère toutefois que les glands de chêne étaient consommés à l'époque classique : en effet, il évoque le goût des glands (Rech. 3, 8, 2-7), qui est, selon lui, un critère qu'utilisent les habitants de Macédoine pour distinguer différentes sortes de chêne.

Le pistachier tend à disparaître des assemblages dès la fin du Néolithique. Ce dernier, principalement représenté par deux espèces (lentisque et térébinthe), est, selon les sources écrites, exploité à l'époque classique pour son huile (Diosc., Pharm. 13, 2). Tout comme le sureau et la ronce, le pistachier et le chêne sont plutôt cueillis et relèvent davantage de l'espace sauvage à l'époque classique. Ainsi les auteurs grecs ne les traitent pas parmi les plantes cultivées.

L'amandier (Prunus dulcis) est également fréquemment attesté au Néolithique, non seulement dans le nord de la Grèce, mais également en Crète et dans le Péloponnèse. Comme le poirier (Pyrus sp.) et le cornouiller mâle (Cornus mas), il pourrait avoir été mis en culture entre le Néolithique et l'époque classique, pour laquelle les textes l'attestent. Malheureusement, peu de données carpologiques peuvent venir étayer cette hypothèse.

Dès le Néolithique, les défrichements nécessaires à l'agriculture ont pu donner lieu à la formation de haies et lisières en bordure des parcelles cultivées, ce qui favorise la croissance d'espèces héliophiles comme le cornouiller, l'amandier, le poirier, la ronce, le sureau, ou l'aubépine. Il s'agit sans doute d'une des premières formes d'arboriculture pérenne (Kreuz, 1992, 1998; Bouby et Ruas, 2014; Valamoti, 2015), mise en évidence par l'association de graines et de charbons de taxons de lisières dans les assemblages carpologiques et anthracologiques, comme ceux provenant du site de Makri (Ntinou et Badal 1998; Ntinou 2002). Lors du défrichement des parcelles, certains arbres ont pu être épargnés : cette forme de culture aurait notamment favorisé la croissance de chênes dans les champs cultivés.

L'entretien de haies afin de favoriser les fruitiers qui y croissent a pu se maintenir jusqu'à l'époque romaine, bien que les preuves soient difficiles à établir. La rareté des restes de ces fruitiers de lisière dans les assemblages carpologiques peut être due aux méthodes de récupération des restes : l'échantillonnage systématique et le recours au tamisage à maille fine sont rares, voire inutilisés, jusqu'aux années 1990 sur bien des sites de Grèce, ainsi les plus petits restes comme ceux de ronce ou de sureau sont peut-être moins représentés. 
Les sources épigraphiques et littéraires de langue grecque ne mentionnent pas de haies, ainsi il est difficile d'attester cette pratique pour l'époque classique où les indices carpologiques sont rares. Toutefois, parmi les taxons précédemment évoqués, certains font partie des arbres les plus couramment cultivés d'après les écrits de Théophraste, et ils apparaissent également dans les baux ruraux : c'est le cas du poirier, peutêtre de l'amandier et du noisetier. En outre, les arbres cultivés sur les terres publiques et sacrées ne sont pas tous dénommés dans les baux : amandier, poirier, chêne pourraient faire partie des " autres arbres", ou des dendra karpofora. Tandis que les recommandations relatives aux plantations de vignes sont parfois très précises (e.g. IG II $^{2} 2493$ : mention des rangs de vigne, de leur espacement et des céréales et légumineuses à y semer), rien n'est dit de la manière dont doivent être plantés les autres arbres : rien n'interdit de supposer qu'ils se trouvent en bordure de parcelle.

\section{Fruitiers introduits}

Certaines espèces semblent apparaitre en Grèce entre l'âge du Bronze et l'époque romaine. Il s'agit tout d'abord du grenadier (Punica granatum) et du noyer (Juglans regia). Tous deux pourraient être introduits au cours de l'âge du Bronze : cette période apparaît comme une période de changements, caractérisée par une intense activité agricole d'après les marqueurs polliniques (Atherden et al., 1993; Lespez, 2012) et probablement par de nouvelles pratiques alimentaires (Valamoti, 2007).

La première mention de grenadier remonte au Bronze ancien. Il s'agit de charbons découverts sur le site d'Akrotiri (3500/3000 avant notre ère, Asouti, 2003). La plus ancienne mention de graine de grenade vient du palais de Tirynthe et remonte au Bronze récent (1350-1050 avant notre ère; Kroll, 1982). Il est possible que les premières tentatives d'acclimatation du grenadier remontent au Bronze ancien et soient restées isolées jusqu'à ce qu'il soit acclimaté et cultivé en Grèce. C'est le cas à l'époque archaïque, comme l'atteste les textes d'Homère (Odyssée 7, 112-116 et 122-126; 24, 245-247; 24, 340-342; Amigues, 2016). A l'époque classique, il en existe peut-être même des formes subspontanées. En effet, ni Théophraste ni Dioscoride ne font référence à son origine exotique, et Dioscoride signale l'existence du grenadier « sauvage " (Diosc. Pharm. 1, 111). Plusieurs baux ruraux de Délos et Rhénée mentionnent le grenadier, toujours unique, bien que les expressions comme « autres arbres " puissent masquer leur présence : cela en fait un arbre cultivé, mais relativement rare.

D'après les données palynologiques, le noyer est absent de Grèce avant le Néolithique. La mention la plus ancienne de pollen de noyer provient de l'environnement du site de
Dispilio en Macédoine occidentale (Néolithique récent, 5300-3500 avant notre ère). On le trouve mentionné par Dioscoride, toutefois il ne fait pas clairement état de sa culture en Grèce, ni de sa présence dans la végétation spontanée.

D'autres taxons sont sans aucun doute introduits en Grèce. Cependant, il reste non seulement très difficile de déterminer la date de leur introduction, d'autre part il est presque impossible de déterminer si ces espèces ont été acclimatées dès l'Antiquité, si elles ont d'abord été importées sous forme de fruits ou d'arbres, à une seule ou à plusieurs reprises. C'est le cas du pêcher (Prunus persica) et du cognassier (Cydonia oblonga), dont les attestations carpologiques sont très rares : un noyau de pêche provenant de Samos (Kučan, 1995, 2000) et un pépin de coing dont l'identification est incertaine (Luce et Marinval, 2008). Ces deux découvertes sont datées du viI ${ }^{\mathrm{e}}$ siècle avant notre ère, ce qui interdit de supposer une introduction avant cette date. Quant au palmier et aux agrumes, seules les sources écrites nous renseignent sur la connaissance que pouvaient en avoir les Anciens, et rien ne permet de déterminer les espèces connues ni si certaines étaient acclimatées en Grèce, même à l'époque impériale.

Ces indices restent minces, toutefois l'apparition de nouvelles espèces, même si elles ne laissent que des vestiges fragmentaires, permet de supposer que l'essor des contacts et des échanges à partir de l'époque archaïque a contribué à une diversification des espèces fruitières.

\section{Les pommiers et autres fruits charnus indéterminés}

Dans les sources écrites, certains termes correspondent à des catégories de fruitiers. Parmi ces catégories, celle des " pommiers " est très souvent mentionnée. Le mot mèléa, complété parfois d'un adjectif ou d'un complément du nom, désigne différents fruitiers. Le fruit de cet arbre est désigné par le terme mêlon, lui aussi accompagné d'un complément. L'examen des noms de fruits formés sur le modèle mêlon accompagné d'un adjectif révèle qu'ils présentent plusieurs caractéristiques communes : il s'agit de fruits charnus, à noyaux ou à pépins. Les fruits dont le nom est formé au moyen du nom mêlon sont pour certains des fruits introduits au cours du $1^{\text {er }}$ millénaire avant notre ère, comme le cognassier ou le pêcher. Il s'agit donc de fruits exotiques, qui ne possèdent pas de nom en grec qui leur soit propre. Toutefois, d'autres sortes de " pommes " apparaissent dans les textes, pour désigner non pas des fruits provenant de contrées lointaines, mais pour nommer des fruits sauvages. Pour ne prendre qu'un exemple, le "faux-abricotier " (Prunus coccomilia) est nommé " pomme de printemps" par les anciens ('̇apıvó - eariná). Il s'agit d'après Théophraste 
(Rech. 2, 1, 3) d'un arbre sauvage; Dioscoride rapproche ce fruit de la pomme sauvage (óyprov - ágrion), également astringent (Diosc. Pharm. 1, 115, 4); enfin le nom que tous lui donnent suggère qu'il s'agit d'un arbre dont les fruits mûrissent tôt.

Ainsi, mêlon apparaît comme un terme bien pratique pour désigner les fruits charnus peu courants, non cultivés ou encore cultivés depuis peu (depuis l'époque romaine). Ces différents " pommiers » cultivés semblent tous avoir un statut équivalent : il s'agit de fruitiers qui offrent du bois, des fruits charnus qui peuvent se consommer frais, séchés ou encore se conserver dans le vinaigre, mais ils ne sont pas source de produits aussi utiles et multiples que la vigne et l'olivier.

Les termes opôra et akrodrua désignent des catégories définies par des critères difficiles à établir, vraisemblablement variables selon les auteurs, et qui, par conséquent regroupent selon ces derniers des fruitiers différents. Il semble qu'ils désignent des taxons autres que la vigne, l'olivier et le figuier et leurs mentions dans les baux ruraux sont le signe d'une diversité cultivée bien plus importante que la triade vigneolivier-figuier. Les expressions comme dendra akrodrya ou dendra opôra nous renseignent avant tout sur la manière dont sont perçus les fruitiers : le choix de désigner des arbres par un terme qui renvoie à la qualité de leurs fruits, à l'usage qui en sera fait ou encore à leur couleur relève d'une logique différente de celle qui conduit à nommer un élément selon des critères d'identification morphologiques, anatomiques et biologiques visant à distinguer une espèce d'une autre. En l'occurrence, ces noms de catégories semblent renvoyer à un ensemble de fruitiers dont l'utilité économique est semblable, et qui les distingue des fruitiers de rendement que sont la vigne, le figuier et l'olivier.

\section{Conclusion}

Bien que cette étude conduise davantage à un ensemble d'hypothèses qu'à une histoire de la diversité fruitière en Grèce protohistorique et antique, elle montre combien la confrontation des sources se révèle efficace pour mettre en lumière plusieurs aspects de l'évolution de cette diversité.

Il est possible de situer les débuts de l'arboriculture fruitière en Grèce au Néolithique, avec la mise en culture de la vigne et du figuier, ainsi que de l'olivier, de l'amandier et du poirier. Les indices en faveur d'une culture sont plus nombreux à partir du Néolithique récent, toutefois l'exploitation de ces fruitiers et la consommation de leurs fruits se laissent deviner dès le Néolithique ancien.

Le cortège des fruitiers exploités évolue entre le Néolithique et l'époque romaine, avec plusieurs périodes de changement.
A partir de l'âge du Bronze, les fruits sauvages comme les mûres de ronces, les baies de sureau, les cornouilles sont plus rares dans les assemblages. Plusieurs biais peuvent expliquer cette raréfaction et on ne peut conclure à leur abandon par les hommes. Toutefois, les changements dans les pratiques alimentaires liés aux changements économiques et culturels de l'âge du Bronze se reflètent peut-être à travers le choix des fruits consommés. De plus, de nouvelles espèces sont introduites à l'âge du Bronze, comme la grenade ou le noyer. Ensuite c'est probablement au cours du premier millénaire, avec l'essor des échanges entre la Grèce et le reste du monde méditerranéen, que sont introduits et acclimatés de nouveaux fruitiers. Dater l'introduction du pêcher, du cognassier ou du cerisier est difficile compte tenu du fait que les données carpologiques sont peu abondantes pour le $1^{\text {er }}$ millénaire, et qu'il y a également peu de données écrites avant l'époque classique qui permettent de documenter cette question.

\section{Remerciements}

L'auteur remercie les deux rapporteurs anonymes dont les commentaires ont largement contribué à améliorer le manuscrit.

Ce travail est issu d'une thèse de doctorat financée par un contrat doctoral de l'Université Paris 1 Panthéon-Sorbonne. Il a été achevé dans le cadre du projet PLANTCULT "Identifying the Food Cultures of Ancient Europe "financé par l'ERC (European Research Council) dans le cadre du programme Horizon 2020 (Grant Agreement no.

682529), Consolidator Grant 2016-2021.

\section{Bibliographie}

\section{Sources littéraires}

Athénée : Atheneus "Deipnosophists ", tome 1, éd. C.B. Gulick, Londres/Cambridge, 1961.

Dioscoride :

Dioscorides De materia medica, trad. L.Y. Beck, Hildesheim, OlmsWeidmann, 2005.

Pedanii Dioscuridis Anazarbei "De Materia Medica " libri quinque, éd. M. Wellmann, Berlin, Weidmann, 1958 (2 édition).

Galien : Claudii Galeni Opera omnia, vol. 6, éd. C.G. Kühn (éd.), Hildesheim, 1965.

Homère : Odyssée, tomes 1 et 3, éd. V. Bérard, Paris, Les Belles Lettres, 1924.

Théophraste :

Recherches sur les plantes, tome 1 et 2, éd. S. Amigues, Paris, Les Belles Lettres, 2003a et b ( $2^{\mathrm{e}}$ tirage). 
Les causes des phénomènes végétaux, tome 1, éd. S. Amigues, Paris, Les Belles Lettres, 2012.

\section{Abréviations des corpus épigraphiques}

Bresson Bresson A. (1991), Recueil des inscriptions de la Pérée rhodienne, Paris, Les Belles Lettres (Annales littéraires de l'Université de Besançon. Centre de Recherches d'Histoire Ancienne), $249 \mathrm{p}$.

EA Epigraphica Anatolica

ID Inscriptions de Délos. 1926-1972

IG Inscriptiones Graecae. 1903 -

$\boldsymbol{I G}^{\mathbf{2}}$ Inscriptiones Graecae, editio minor. 1913 -

I. Mylasa I. Blümel W. (1987), Die Inschriften von Mylasa. Teil I : Inschriften der Stadt, Bonn, R. Habelt (Inschriften griechischer Städte aus Kleinasien), 271 p.

I. Mylasa II. Blümel W. (1988), Die Inschriften von Mylasa. Teil II : Inschriften aus der Umgebung der Stadt, Bonn, R. Habelt (Inschriften griechischer Städte aus Kleinasien), 225 p.

IThesp Roesch P. (2009), Inscription de Thespies. Fascicule 2, IThesp 44-87, édition électronique mise en forme et publié par Gilbert Argoud, Albert Schachter et Guy Vottero, Lyon.

Nouveau choix Institut Fernand Courby (2005), Nouveau choix d'inscriptions grecques, Paris, Les Belles Lettres (Epigraphica), $242 \mathrm{p}$.

Sinuri Robert L. (1945), Le Sanctuaire de Sinuri près de Mylasa. Première partie: Les inscriptions grecques, Paris, De Boccard (Mémoires de l'Institut francais d'archéologie de Stamboul), 119 p.

\section{Articles et ouvrages}

Amigues, S., 1999. Les traités botaniques de Théophraste. In G. Wöhrle (dir.). Geschichte der Mathematik und der Naturwissenschaften in der Antike Band 1 : Biologie. Steiner, Stuttgart, 124-154.

Amigues, S. (éd.), 2003a. Théophraste. Recherches sur les plantes. Livres 1 et 2, Collection des Universités de France. Les Belles Lettres, Paris.

Amigues, S. (éd.), 2003b. Théophraste. Recherches sur les plantes. Livres 3 et 4, Collection des Universités de France. Les Belles Lettres, Paris.

Amigues, S. (éd.), 2012. Théophraste. Les causes des phénomènes végétaux. Tome 1, Collection des Universités de France. Les Belles Lettres, Paris.

Amigues, S., 2016. Littérature gréco-latine et arbres fruitiers de l'Antiquité. Introduction et mise en culture. In M.-P. Ruas (dir.). Des fruits d'ici et d'ailleurs. Regards sur l'histoire de quelques fruits consommés en Europe. Omniscience, Montreuil, 153-168.

Amouretti, M.-C., Brun, J.-P., (dir.) 1993. La production du vin et de l'huile en Méditerranée, Bulletin de Correspondance Hellénique, supplément 26. De Boccard, Paris.

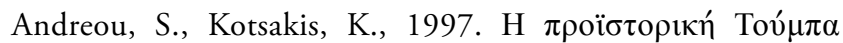

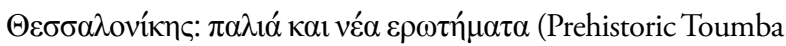
Thessalonikis: old and new questions). Archaiologiko Ergo sti Makedonia kai Thraki, 11a : 369-387.

Asouti, E., 2003. Wood charcoal from Santorini (Thera): new evidence for climate, vegetation and timber imports in the Aegean Bronze Age. Antiquity, 77 : 471-484.

Atherden, M., Hall, J.R., Wright, J.C., 1993. A pollen diagram from northeast Peloponnese, Greece: implication for vegetation history and archaeology. The Holocene, 3 : 351-356.

Badal, E., Ntinou, M. 2013. Wood charcoal analysis: the local vegetation. In N. Efstratiou, A. Karetsou, M. Ntinou (dir.). The Neolithic Settlement of Knossos in Crete. New evidence for the early occupation of Crete and the Aegean Islands. INSTAP Academic Press, Philadelphie, 119-132.

Becker, C., Kroll, H., 2008. Das prähistorische Olynth. Ausgrabungen in der Toumba Aghios Mamas 1994-1996, Ernährung und Rohstoffnutzung im Wandel, Prähistorische Archäologie in Südeuropa 22. Verlag Marie Leidorf, Berlin.

Bendall, L.M., 2007. Economics of religion in the Mycenaean World, Oxford University School of Archaeology Monograph 67. Oxford University School of Archaeology, Oxford.

Bennett, E.L., 1958. The olive oil tablets of Pylos, Minos, Revista de Filologia Egea, Suppl. 2. Universidad de Salamanca, Salamanque.

Bennett, E.L., Olivier, J.-P. (dir.). 1973. The Pylos tablets transcribed. Part 1, texts and notes, Incunabula graeca (Roma). Edizioni dell'Ateneo, Rome.

Bennett, E.L., Olivier, J.-P. (dir.). 1976. The Pylos tablets transcribed. Part 2, Hands, concordances, indices, Incunabula graeca (Roma). Edizioni dell'Ateneo, Rome.

Blitzer, H., 1993. Olive cultivation and oil production in Minoan Crete. In M.-C. Amouretti, J.-P. Brun (dir.). La Production Du Vin et de L'huile En Méditerranée, Bulletin de Correspondance Hellénique, Supplément 26. De Boccard, Paris, 163-175.

Boissinot, P., 2013. Archéologie des vignobles grecs antiques. Food \& History, $11: 113-124$.

Bookidis, N., Hansen, J., Snyder, L.D., Goldberg, P., 1999. Dining in the Sanctuary of Demeter and Kore at Corinth. Hesperia, $68: 1-54$.

Bottema, S., Sarpaki, A., 2003. Environnemental change in Crete: a 9000-years of Holocene vegetation history and the effect of the Santorini eruption. The Holocene, 13 : 733-749.

Bouby, L., Ruas, M.-P., 2014. Adding diversity. Between occasional food and speculative productions: diversity of fruit uses, diversity of practices regarding fruit tree cultivation (Introduction). In A. Chevalier, E. Marinova, L. Peña-Chocarro (dir.). Plants and People. Choices and diversity through time, EARTH 1. Oxbow Books, Oxford, 141-149.

Brogan, T., Morrison, J.E., Sofianou, C., Mylona, D., Margaritis, E., 2013. Living off the fruits of the sea: new evidence for

ArcheoSciences, revue d'archéométrie, 43(1), 2019, p. ??-?? 
dining at Papadiokampos. In S. Voutsaki, S.M. Valamoti, (dir.). Diet, Economy and Society in the Greek World. Towards a Better Integration of Archaeology and Science. Proceedings of the International Conference Held at the Netherlands Institute at Athens on 22-24 March 2010, Pharos Supplément 1. Peeters, Liège, 123-131.

Brun, J.-P., 2003. Le vin et l'huile dans la Méditerranée antique. Errance, Paris.

Brun, J.-P., 2004. Archéologie du vin et de l'huile. De la préhistoire à l'époque hellénistique. Errance, Paris.

Bruneau, P., Fraisse, P., 1981. Un pressoir à vin à Délos. Bulletin de Correspondance Hellénique, 105 : 127-173.

Brunet, M., 1990. Contribution à l'histoire rurale de Délos aux époques classiques et hellénistiques. Bulletin de Correspondance Hellénique, 114 : 669-682.

Chadwick, J., 1976. The Myceanean World. Cambridge University Press, Cambridge.

Chevalier, J., Gheerbrant, A., 1982. Dictionnaire des symboles. Robert Laffont, Paris.

Colaianni, G., Fiorentino, G., Fouache, E., Pontrandolfo, A., Santoriello, A., Scelzo, F., 2012. Palaeoenvironmental reconstruction of the Krios valley, Northern Achaea (Greece): archaeobotanical analysis conducted as part of the Aegalia Survey Project. ArcheoScience, 36 : 127-137.

Colledge, S., 2001. Plant exploitation on Epipalaeolithic and Early Neolithic sites in the Levant, BAR International Series 986. Archaeopress, Oxford.

Couplan, F., 1989. Le régal végétal. Plantes sauvages comestibles, Encyclopédie des plantes comestibles d'Europe. Equilibres aujourd'hui, Flers.

Duhoux, Y., 1993. Les premiers phytonymes grecs: les données mycéniennes. In Actes Du Colloque International "Les Phytonymes Grecs et Latins ", Nice, 14-16 Mai 1992. Publication du centre LAMA, Nice, 99-122.

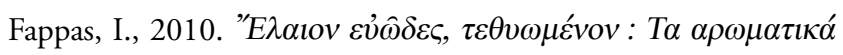

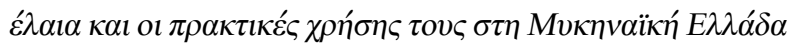

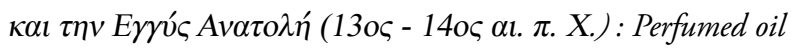
and practices of use in Mycenean Greece and the ancient Near East (13st-14st century BC). Kritiki Estia, Chania.

Fiorentino, G., Solinas, F., 2006. Carboni e carporesti dal palazzo di Monastiraki. In A. Kanta, M. Marazzi (dir.). Monastiraki 1, Missione Monastiraki: Campagne 2002/2004. Suor Orsola Benincasa nell'Università, Naples, 123-140.

Follieri, M., 1979. Provviste alimentari vegetali da una casa minoica ad Hagia Triada (Creta). Annuario della Scuola Archeologica di Atene e delle Missioni Italiane in Oriente, 41-42 : 165-172.

Garnier, N., Valamoti, S.M., 2016. Prehistoric wine making at Dikili Tash (Northern Greece): integrating residue analysis and archaeobotany. Journal of Archaeological Science, 74 : 195-206.
Gilman, A., 1981. The development of Social stratification in Bronze Age Europe. Current Anthropology, 22 : 1-23.

Halstead, P., 1999. Towards a model of Mycenaean Palace mobilization. In M.L. Galaty, W.A. Parkinson (dir.). Rethinking Mycenaean Palaces: New Interpretations of an Old Idea. Cotsen Institute of Archaeology, Los Angeles, 35-41.

Halstead, P., 2011. Redistribution in Aegean Palatial societies: terminology, scale and significance. American Journal of Archaeology, $115: 229-235$.

Halstead, P., Jones, G., 1980. Early Neolithic economy in Thessalysome evidence from excavations at Prodromos. Anthropologica, 1 : 93-117.

Hamilakis, Y., 1996. Wine, Oil and the dialectics of power in Bronze Age Crete : a review of the evidence. Oxford Journal of Archaeolgy, $15: 1-32$.

Hansen, J., 1991. The palaeoethnobotany at Franchthi Cave, Excavation at Franchthi cave, Greece. Indiana University Press, Bloomington.

Hansen, J.M., Allen, S.E., 2011. Palaeoethnobotany. In D.J. Pullen (dir.). The Early Bronze Age Village on Tsoungiza Hill, The Nemea Valley Archaeological Project. The American School of Classical Studies at Athens, Athènes, 805-891.

Hopf, M., 1961. Pflanzenfunde aus Lerna/Argolis. Der Züchter, 31 : 239-247.

Hopf, M., 1962a. Nutzpflanzen vom lernäischen Golf. Jahrbuch des Römisch-Germanischen Zentralmuseums Mainz, 9, 1-19.

Hopf, M., 1962b. Bericht über die Untersuchung von Samen und Holzkohlenresten von der Argissa-Magula aus den Präkeramischen bis Mittelbronzenzeitlichen Schichten. In V. Milojčić (dir.). Die Deutschen Ausgrabungen auf der ArgissaMagula in Thessalien 1.Das präkeramische Neolithikum sowie die Tier-und Pflanzenreste, Beiträge zur Ur-und Frühgeschichtlichen Archäologie des Mittelmeer-Kulturraumes. R. Habelt, Bonn, 101119.

Jones, G., 1982. Cereal and pulse remains from Protogeometric and Geometric Iolkos, Thessaly. Anthropologika, 3 : 75-78.

Jones, G., 1984. The late Minoan II plants remains. In M. Popham (dir.). The Minoan Unexplored Mansion, The British Scool at Athens, Supplementary volume 17. Londres, 303-306.

Jones, G., 1995. Charred grains from Late Bronze age Gla, Boiotia. Annual of the British School at Athens, 90 : 235-238.

Jones, G., Halstead, P., 1993. Charred plant remains from NeotithicBronze Age Plateia Magoula Zarkou, Thessaly. Annual of the British School at Athens, 88 : 1-5.

Kent, J.H., 1948. The temple estates of Delos, Rheneia ans Mykonos. Hesperia, 117 : 243-338.

Killen, J.T., 1999. Some observations on the new Thebes tablets. Bulletin of the Institute of Classical Studies, 43 : 217-219.

Killen, J.T., 2004. Wheat, Barley, Flour, Olives and Figs on Linear B tablets. In P. Halstead, J.C. Barrett (dir.). Food, Cuisine and 
Society in Prehistoric Greece. Sheffield Academic Press, Sheffield, 155-173.

Killen, J.T., Olivier, J.-P., 1989. The Knossos tablets, 5e édition. Universidad de Salamanca, Salamanque.

Kotzamani, G., Livarda, A., 2017. Archaeobotanical Remains. In R.A.K. Smith, M.K. Dabney, E. Pappi, S. Triantaphyllou, J.C. Wright (dir.). Ayia Sotira. A Mycenean Chamber Tomb Cemetery in the Nemea Valley, Greece. INSTAP Academic Press, Philadelphia, 139-145.

Kraft, J.C., Rapp, G.R.J., Ashenbrenner, S.E., 1980. Late Holocene Palaeogeomorphic reconstructions in the area of the Bay of Navarino: Sandy Pylos. Journal of Archaeological Science, 7 : 187-210.

Kreuz, A., 1992. Charcoal from ten Early Neolithic settlements in central Europe and its interpretation in terms of woodland management and wildwood resources. Bulletin de la Société Botanique de France, 139 : 383-394.

Kreuz, A., 1998. Closed forest or open woodland as natural vegetation in the surroundings of Linearbandkeramik settlements? Vegetation History and Archaeobotany, 17 : 51-64.

Kroll, H., 1979a. Kulturpflanzen aus Dimini. In U. Körber-Grohne (dir.). Festschrift Maria Hopf, Archaeophysika 8, 173-189.

Kroll, H., 1979b. Pflanzliche Grossreste vom Siedlungshügel bei Kastanas. Jahrbuch des Römisch-Germanischen Zentralmuseums Mainz, 26 : 229-239.

Kroll, H., 1981. Thessalische Kulturpflanzen. Zeitschrift für Archäologie, 15 : 97-103.

Kroll, H., 1982. Kulturpflanzen von Tiryns. Archäologischer Anzeiger, 1982, (3) : 467-485.

Kroll, H., 1983. Die Planzenfunde: Kastanas. Ausgrabungen in einem Siedlungshügel der Bronze- und Eisenzeit Makedoniens 19751979. V. Spiess, Berlin.

Kroll, H., 1984. Zum Ackerbau gegen Ende des Mykenischen Epoche in der Argolis. Archäologischer Anzeiger 1984 : 211-222.

Kroll, H., 1993. Kulturpflanzen von Kalapodi. Archäologischer Anzeiger $11: 161-182$.

Kroll, H., 2003. Rural Plenty: the result of hard work - Rich Middle Bronze Age plant remains from Aghios Mamas, Chalkidike. In G.A. Wagner, E. Pernicka, H.P. Uepermann (dir.). Troia and the Troad. Scientific Approaches. Springer, Berlin, 293-301.

Kučan, D. 1995. Zur Ernährung und dem Gebrauch von Pflanzen im Heraion von Samos im 7. Jahrhundert v. Chr. Jahrbuch des Deutschen Archäologischen Instituts, 110 : 1-64.

Kučan, D. 2000. Rapport synthétique sur les recherches archéobotaniques dans le sanctuaire d'Héra de l'île de Samos. Pallas, 54 : 99-108.

Launey, M., 1937. Le verger d'Héraklès à Thasos. Bulletin de Correspondance Hellénique, 61 : 380-409.

Lespez, L., 2012. Les temps de l'environnement et des paysages des systèmes fluviaux au cours de l'Holocène (Normandie, Grèce, Mali).
Mémoire d'habilitation à diriger les recherches, Université de Caen, Caen.

Lieutaghi, P., 2004. Le livre des arbres, arbustes et arbrisseaux, 2e éd. Actes Sud, Paris.

Livarda, A., Kotzamani, G., 2013. The archaeobotany of Neolithic and Bronze Age Crete: synthesis and prospects. Annual of the British School at Athens, 108 : 1-29.

Luce, J.-M., Marinval, P., 2008. L'alimentation végétale. In J.-M. Luce (dir.). Fouilles de Delphes II, Topographie et architecture 13. L'aire du pilier des Rhodiens (fouilles 1990-1992). À la frontière du profane et du sacré. De Boccard, Paris, 230-256.

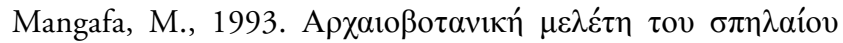

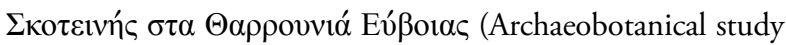
of the Skoteini Cave at Tharrounia, Euboea). In A. Sampson (dir.). Skoteini Tharrounia. Athènes, 360-369.

Mangafa, M., Kotsakis, K., Andreou, S., 1998. А $\mu \pi \varepsilon \lambda о \kappa \alpha \lambda \lambda \iota \varepsilon ́ p \gamma \varepsilon ı \alpha$

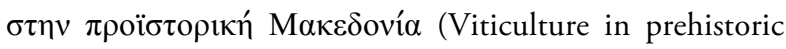
Macedonia: evidence from prehistoric Toumba Thessalonikis).

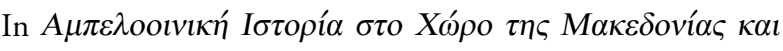
$\tau \eta \varsigma \Theta \rho \alpha ́ \kappa \eta \varsigma$ (History of Viticulture and Wine in Macedonia and Thrace), ETBA, Athènes, 158-169.

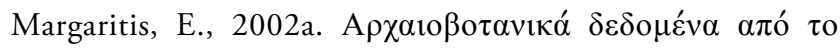

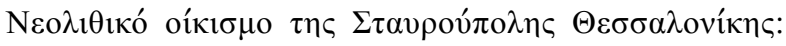

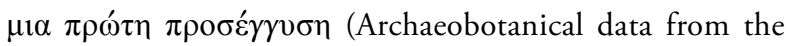
Neolithic settlement of Stavroupoli Thessalonikis: a first approach). In D. Grammenos, E. Kotsos (dir.). $\Sigma \omega \sigma \tau \imath \kappa \varepsilon ́ \varsigma$

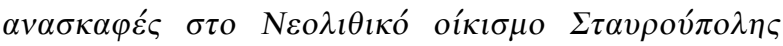
$\Theta \varepsilon \sigma \sigma \alpha \lambda$ ovíkn (Rescue excavations at the Neolithic settlement of Stavroupoli Thessalonikis). Archaiologiko Institouto Boreias Elladas, Thessalonique, 805-824.

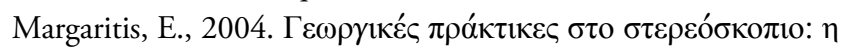

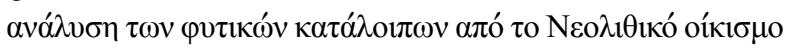

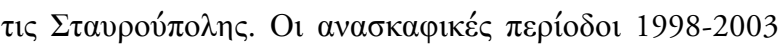
(Agricultural practices under the microscope: the analysis of the plant remains from the Neolithic settlement of Stavroupoli. The excavations 1998-2003). In D. Grammenos, E. Kotsos (dir.).

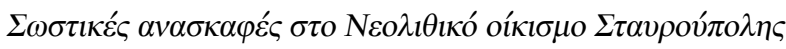
$\Theta \varepsilon \sigma \sigma \alpha \lambda o v i ́ \kappa \eta \varsigma$ (Rescue excavations at the Neolithic settlement of Stavroupoli Thessalonikis). Archaiologiko Institouto Boreias Elladas, Thessalonique, 605-612.

Margaritis, E., 2013a. Annexe 3 : Archaeobotanical analyses. Seeds assembalges from the refuse pit Fo221. In S. Verdan (dir.). Le Santuaire d'Apollon Daphnephoros à l'époque Géometrique, Eretria 22. Ecole Suisse d'Archéologie, Athènes, 265-269.

Margaritis, E., 2013b. Foodstuffs, fruit trees, cultivation and occupation patterns at Dhaskalio. In C. Renfrew, O. Philaniotou, N. Brodie, G. Gavalas, M.J. Boyd (dir.). The settlement at Dhaskalio. The Sanctuary on Keros and the origins of Aegean ritual practices: the excavations of 2006-2008. MacDonald Insitute for Archaeological Research, Cambridge, 389-404. 
Margaritis, E., 2014a. Acts of destruction and acts of preservation: plants in the ritual landscape of Prehistoric Greece. In G. Touchais, R. Laffineur, F. Rougemont (éds.). Physis. L'environnement Naturel et La Relation Homme-Milieu Dans Le Monde Ééen Protohistorique. Actes de la 14e Rencontre Égéenne Protohistorique, Paris, Institut Nationale d'Histoire de l'Art (INHA),11-14 Décembre 2012, Aegeum 27. Peeters, Liège, 279-285.

Margaritis, E., 2014b. The kapeleio at Hellenistic Krania. Food consumption, disposal and the use of space. Hesperia, 83 : 103-121.

Margaritis, E., 2015. Cultivating Classical Archaeology: Agricultural activities, uses of space and occupation patterns in Hellenistic Greece. In D.C Haggis, C.M. Antonaccio (dir.). Classical archaeology in context: theory and practice in excavation in the Greek world. De Gruyter, Berlin/Boston, 333-354.

Margaritis, E., 2016. Agricultural production and domestic activities in rural hellenistic Greece. In E.M. Harris, D.M. Lewis, M. Woolmer (dir.). The Ancient Greek economy. Markets, households and city-states. Cambridge University Press, Cambridge, 187-204.

Margaritis, E., Jones, M.K., 2006. Beyond cereals: crop-processing and Vitis vinifera L. Ethnography, experiment and charred grape remains from Hellenistic Greece. Journal of Archaeological Science, 33 : 784-805.

Megaloudi, F., 2005. Burnt sacrificial plant offering from Hellenistic time: an archaeobotanical case of study from Messene, Peloponese, Greece. Vegetation History and Archaeobotany, 14 : 329-340.

Megaloudi, F., 2006. Plants and diet in Greece from Neolithic to Classic Periods: the archaeobotanical remains, BAR International Series 1516. Archaeopress, Oxford.

Megaloudi, F., 2008. Sarakenos cave: archaeobotanical remains from the LN levels. In A. Sampson (dir.). Sarakenos cave at Akraephnion, Boeotia, Greece, vol. 1 : the Neolithic and the Bronze Age. University of Aegean, Athènes, 424-428.

Megaloudi, F., 2016. Counting fruits and nuts from historical deposits of Greece (900-300 BC). Archaeobotanical data and literary evidence. In M.-P. Ruas (dir.). Des fruits d'ici et d'ailleurs. Regards sur l'histoire de quelques fruits consommés en Europe. Omniscience, Montreuil, 141-151.

Megaloudi, F., Marinval, P., 2002. Données préliminaires sur l'économie végétale du site de Ftelia, Cyclades (Grèce) au Néolithique récent. In A. Sampson (dir.). The Neolithic Settlement at Ftelia, Mykonos. University of Aegean, Rhodes, 191-200.

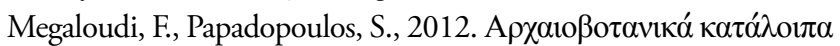

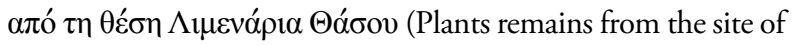
Limenaria, Thasos), in S. Papadopoulos, D. Malamidou (dir.).

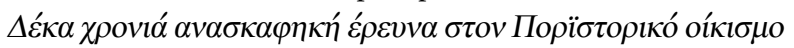

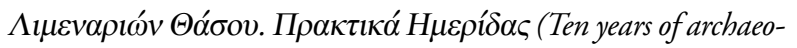
logical research at the prehistoric settlement of Limenaria, Thasos). Thessalonique, 67-76.
Megaloudi, F., Papadopoulos, S., Sgourou, M., 2007. Plant offerings from the classical necropolis of Limenas, Thasos, Northern Greece. Antiquity, 81 : 933-943.

Melena, J.L., 1983. Olive oil and other sorts of oil in Mycenean tablets. Minos, $18: 89-123$.

Miller, N.F., 2008. Sweeter than wine? The use of the grape in early Western Asia. Antiquity, 82 : 937-946.

Moody, J., Rackham, O., Rapp, G.R.J., 1996. Environmental archaeology of prehistoric north-west Crete. Journal of Field Archaeology, 23 : 273-397.

Ntinou, M., 2002. Vegetation and human communities in Prehistoric Greece. Saguntum - Extra, 5 : 91-103.

Ntinou, M., 2013. Wood charcoal: Vegetation and the use of timber at Dhaskalio. In C. Renfrew, O. Philaniotou, N. Brodie, G. Gavalas, M.J. Boyd (dir.). The settlement at Dhaskalio. The Sanctuary on Keros and the origins of Aegean ritual practices: the excavations of 2006-2008. MacDonald Insitute for Archaeological Research, Cambridge, 417-428.

Ntinou, M., Badal, E., 1998. Charcoal analysis in Neolithic Makri. Saguntum, 31 : 51-54.

Pagnoux, C., 2017. Investigating the introduction of citrus fruit in the Western Mediterranean according to ancient Greek and Latin texts. In V. Zech-Matterne, G. Fiorentino (dir.). Agrumed: Archaeology and history of citrus fruit in the Mediterranean: acclimatization, diversifications, uses. Ecole française de Romel CNRS, Paris, 95-104.

Pagnoux, C., Coubray, S., Zech-Matterne, V., 2016. L'introduction des agrumes en Méditerranée occidentale : données archéobotaniques, iconographiques et littéraires. Food \& History, 14 : 107-129.

Palmer, R., 1994. Wine in the Mycenaean economy, Aegeum 10. Peeters, Liège.

Palmer, R., 1996. Wine and Viticulture in the Linear A and B texts of the Bronze Age Aegean. In P. McGovern, S.J. Fleming, S.H. Katz (dir.). The Origins and Ancient History of Wine. Gordon and Breach, Amsterdam, 269-285.

Pavlopoulos, K., Theodorakopoulou, K., Hayden, B., Tsourou, T., Triantaphyllou, M., Kouli, K., Basiakos, I., Vandarakis, D.,

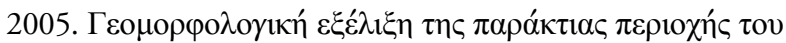

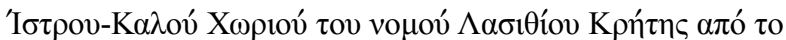

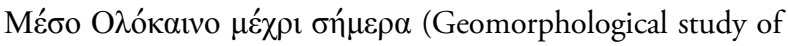
the area of Istron-Kalon Chorion, department of Lassithi, Crete, from mid-Holocene to the present). Bulletin of the Geological Society of Greece, 38 : 54-69.

Pernin, I., 2014. Les baux ruraux dans la Grèce ancienne. Corpus épigraphique et étude. Maison de l'Orient et de la MéditerranéeJean Pouilloux, Lyon.

Philippa-Touchais, A., Touchais, G., Decavallas, O., Gardeisen, A., Ghilardi, M., Margaritis, E., Metaxas, O., Triandaphyllou, S., Tsiolaki, E., 2014. Environnement, alimentation, hyginène et 
mode de vie dans la Grèce mésohelladique : le cas de l'aspis d'Argos. In G. Touchais, F. Rougemont, R. Laffineur (éds.). Physis : l'environnement et la relation homme-milieu dans dans le monde égéen protohistorique. Actes de la 14 e rencontre égénne internationale, Paris, Institut Nationale d'Histoire de l'Art (INHA), 11-14 décembre 2012, Aegeum 27. Peeters, Liège, 531-538.

Poulaki, E., 2003. Farmhouses in the area of Phila-Herakleio and Leivithra of the Macedonian Olympus. In P. Adam-Veleni, E. Poulaki, K. Tzanavari (dir.). Ancient country houses on modern roads. Archaeological Receipts Fund, Athens, 63-70.

Renfrew, C., 1972. The Emergence of Civilisation. The Cyclades and the Aegean in the Third Millennium BC. Oxbow Books, Oxford.

Renfrew, J.M., 1966. A report on recent finds of carbonized cereal grains and seeds from Prehistoric Thessaly. Thessalika, 5 : 21-36.

Renfrew, J.M., 1968. The Cereal Remains. Appendix 10. In J.C. Evans, C. Renfrew (dir.). Excavations at Saliagos near Antiparos, The British Scool at Athens, Supplementary Volume, 5. Londres, 139-141.

Renfrew, J.M., 1969. Palaeobotany and the Neolithic period in Greece and Bulgaria, Thèse de Doctorat, Cambridge University, Cambridge.

Renfrew, J.M., 1972. The plant remains. In P. Warren (dir.). Myrtos. An Early Bronze Age settlement in Crete, British School at Athens, Supplementary volume, 7. Londres, 315-317.

Renfrew, J.M., 1989. Carbonized grains and seeds. In M. Gimbutas, D.M. Shimabuku, S.M.M Winn (dir.). Achilleion: a Neolithic settlement in Thessaly, Greece, 6400-5600 BC. Institute of Archaeolgy, University of California Press, Los Angeles, 388.

Renfrew, J.M., 1997. Plant husbandry at Prehistoric Dimitra (Appendix 14). In D. Grammenos (dir.). Neolithiki Makedonia. Athènes, 220-225.

Renfrew, J.M., 2003. Grains, seeds and fruits from Prehistoric Sitagroi. In E.S. Elster, C. Renfrew (dir.). Prehistoric Sitagroi: Excavations in Northern Greece, 1968-1970, vol. 2: the final report. Institute of Archaeology, University of California Press, Los Angeles, 3-29.

Rougemont, F., 2008. Les enregistrements d'huile de la série Fh de Cnossos. Essai d'interprétation et de comparaison avec les données du bureau de l'huile à Mari. In A. Sacconi, M. Del Freo, L. Godart, M. Negri (dir.). Colloquium Romanum. Atti del XII Colloquio internazionale di Micenologia (Rome, 20-25 février 2006). Fabrizio Serra, Rome/Pise, 669-689.

Ruas, M.-P., 1996. Eléments pour une histoire de la fructiculture en France : données archéobotaniques de l'Antiquité au XVIIe siècle. In M. Colardelle (dir.). L'homme et la nature au MoyenAge, 5 e colloque international de la société d'archéologie médiévale, Grenoble 1993. Errance, Paris, 92-105.

Ruas, M.-P., 2016. Introduction. In M.-P. Ruas (dir.). Des fruits d'ici et d'ailleurs. Regards sur l'histoire de quelques fruits consommés en Europe. Omniscience, Montreuil, 11-38.
Ruas, M.-P., Bouby, L., Pradat, B., 2005. Les restes de fruits dans les dépôts archéologiques du Midi de la France ( $v^{e}-x_{I v} v^{e}$ s.). Archéologie du Midi Médiéval 23-24 : 145-193.

Sarpaki, A., 1992. A palaeoethnobotanical study of the West House, Akrotiri, Thera. Annual of the British School at Athens, 87 : 219230.

Sarpaki, A., 1995. Toumba Balomenou, Chaeronia: plant remains from the Early and Middle Neolithic levels. In H. Kroll, R. Pasternak (éds.). Res archaeobotanicae. Proceedings of the ninth Symposium IWGP, 1992, Kiel. Oetker-Voges-Verlag, Kiel, 281300.

Sarpaki, A., 1999. The archaeobotanical study of Tzambakas House, Rethymon, Crete. In G. Tzedakis, H. Martlew (dir.). Minoans and Mycenaeans, flavours of their time. Kapon, Athènes, 40-41.

Sarpaki, A., 2012. Revisiting the visibility of the grape, grapeproducts, by-products and some insights of its organization from the Prehistoric Aegean, as guided by new evidence from Monastiraki, Crete. IANSA, $3: 211-220$.

Sarpaki, A., 2013. The Economy of Neolithic Knossos: The Archaeobotanical Data. In N. Efstratiou, A. Karetsou, M. Ntinou (dir.). The Neolithic Settlement of Knossos in Crete. New Evidence for the Early Occupation of Crete and the Aegean Islands. INSTAP Academic Press, Philadelphia.

Sarpaki, A., Asouti, E., 2008. A glimpse through a kitchen key-hole at Late Bronze Age Akrotiri: the organic remains. In N. Brodie, G. Gavalas, C. Renfrew (dir.). Horizon: a colloquium on the Prehistory of the Cyclades. MacDonald Insitute for Archaeological Research, Cambridge, 370-376.

Sarpaki, A., Bending, J., 2004. Archaeobotanical assemblages. In J.S. Soles, C. Davaras (dir.). Mochlos IC. Period III. Neopalatial settlement on the coast: the Artisans' Quarter and the farmhouse at Chalinoumouri: the small finds. INSTAP Academic Press, Philadelphia, 126-137.

Sarpaki, A., Kanta, A., 2011. Monastiraki in the Amari region in Crete: some interim archaeobotanical insights into Middle Bronze Age subsistence. In M. AndreadakiVladaki, E. Papadopoulou (éds.), Proceedings of the 10th International Cretological Congress, October 168, 2006, Chania. Chrysostomos, Chania, 247--270.

Shay, J.M., Shay, C.T., 1978. Modern vegetation and fossil plant remains. In G. Rapp, S.E. Ashenbrenner (dir.)., Excavations at Nichoria in Southwest Greece, Vol. 1 : Site, environs and techniques. The University of Minnesota Press, Minneapolis, 41-51.

Shay, C.T., Shay, J.M., 2000. The Charcoal and Seed Remains from Iron Age Kommos. In J.W. Shaw, M.C. Shaw (dir.). Kommos IV. The Greek sanctuary, Part. 1. Princeton University Press, Princeton, 647-668.

Shay, C.T., Shay, J.M., Frego, K.A., Zwiazek, J., 1995. The Charcoal and Seeds from Bronze Age deposits at Kommos. In J.W. Shaw, M.C. Shaw (dir.). Kommos I. The Kommos Region

ArCheoSCIEnCES, revue d'archéométrie, 43(1), 2019, p. ??-?? 
and Houses from the Minoan Town, Part 1: The Kommos region, Ecology and Minoan Industries. Princeton University Press, Princeton, 116-162.

Shay, C.T., Shay, J.M., Kapinga, M.R.M., 1998. Appendix IV: The Bronze Age plant and insect remains and modern vegetation. In $\mathrm{G}$. Walberg (dir.). Excavations on the acropolis of Midea. The excavations on the Lower Terraces 1985-1991, vol. I:1, Skrifter utgivna av Svenska institutet i Athen. Series prima in $4^{\circ}$, Stockholm, 295-357.

Soles, J.S. 2003. The Chalinomouri Farmhouse, in J.S Soles, T.M. Brogan (dir.). Mochlos IA. Period III. Neopalatial settlement on the coast: the Artisans' Quarter and the farmhouse at Chalinoumouri: the small finds, Philadelphia, INSTAP Academic Press (Prehistory Monographs), pp. 103-125.

Treuil, R., Darcque, P., Poursat, J.-C., Touchais, G., 2008. Les civilisations égéennes du Néolithique et de l'âge du Bronze, 2e éd. Presses Universitaires de France, Paris.

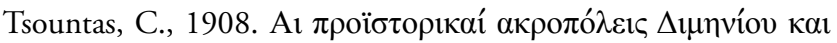
$\Sigma \varepsilon ́ \sigma \kappa \lambda o v$ (The Neolithic acropolis of Dimini and Sesklo). Athènes.

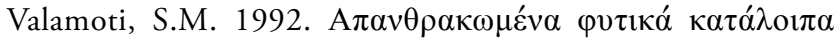

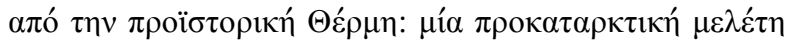
(Charred plant remains from prehistoric Thermi: a preliminary examination, in Greek). Makedonika, 18 : 443-455.

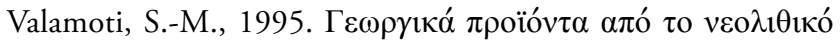

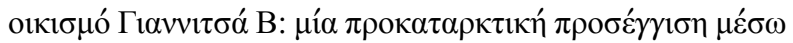
$\tau \omega \nu \alpha \rho \chi \alpha \iota \beta о \tau \alpha \nu \iota \kappa \omega ́ v \delta \varepsilon \delta$ o $\mu \varepsilon ́ v \omega v$ (Agricultural products from the Neolithic settlement of Giannitsa B: a preliminary approach through the archaeobotanical evidence). Archeologiko Ergo sti Makedonia kai Thraki, 6 : 177-184.

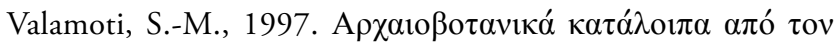

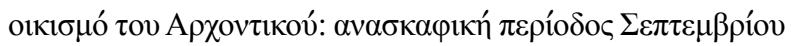
1993 (Archaeobotanical remains from Archondiko: excavation season September 1993). Archeologiko Ergo sti Makedonia kai Thraki, $7: 155-158$.

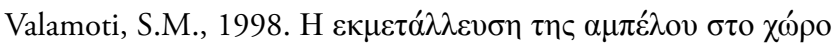

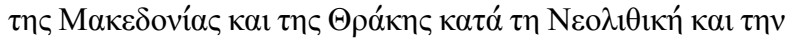

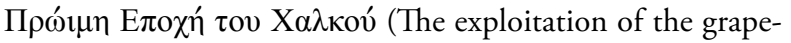
vine in Macedonia and Thrace during the Neolithic and the

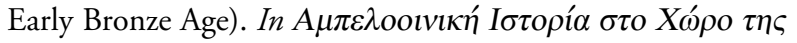

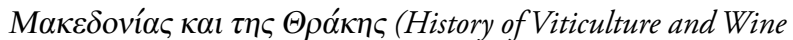
in Macedonia and Thrace). ETBA, Athènes, 137-149.

Valamoti, S.-M., 1999. Charred plant remains from Makriyalos: preliminary observations. In P. Halstead (dir.). Neolithic Society in Greece. Sheffield Academic Press, Sheffield, 136-138.

Valamoti, S.-M., 2004. Plants and People in Late Neolithic and Bronze Age Northern Greece. An archaeobotanical investigation, BAR International Series 1258. Archaeopress, Oxford.

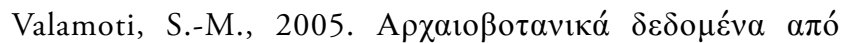

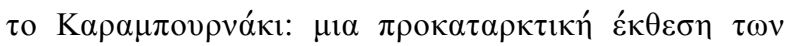

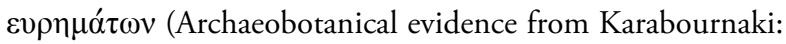
a preliminary examination of the finds). Archeologiko Ergo sti Makedonia kai Thraki, 17 : 201-204.

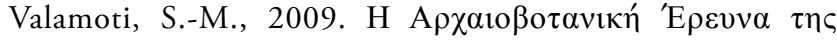

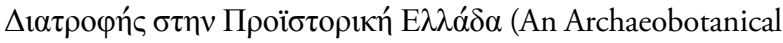
Investigation of Prehistoric Diet in Greece). University Studio Press, Thessalonique.

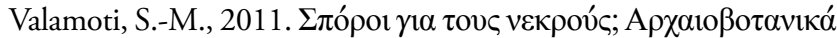

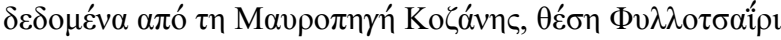
(Seed for the dead? Archaeobotanical remains from MavropigiFyllotsairi in Kozani). In Proceedings of the 1st Archaeological Conference on Archaeological Investigations in Upper Macedonia. Archaeological Museum of Aiani, Kozani, 245-256.

Valamoti, S.M., 2015. Harvesting the "wild"? Exploring the context of fruit and nut exploitation at Neolithic Dikili Tash, with special reference to wine. Vegetation History and Archaobotany, 24 : 35-46.

Valamoti, S.M., Darcque, P., Koukouli-Chrysanthaki, C., Malamidou, D., Tsirtsoni, Z., 2015. An Archaeobotanical investigation of Prehistoric grape vine exploitation and wine making in Northern Greece: recent finds from Dikili Tash. In A. Diler, K. Senol, Ü. Aydinoğlu, (éds.). Olive oil and wine production in Eastern Mediterranean during Antiquity. International Symposium Proceedings 17-19 november 2011, Urla, Turkey. Ege Üniversitesi, Izmir, 125-139.

Valamoti, S.M., Gkatzogia, E., Ntinou, M., 2018. Did Greek colonisation bring olive growing to the North? An integrated archaeobotanical investigation of the spread of Olea europaea in Greece from the 7th to the 1st millennium BC. Vegetation History and Archaeobotany, 27 : 177-195.

Valamoti, S.M., Mangafa, M., Koukouli-Chrysanthaki, C., Malamidou, D., 2007. Grape-pressings from northern Greece: the earliest wine in the Aegean ? Antiquity, 81 : 54-61.

Van Zeist, W., Bottema, S. 1971. Plant husbandry in Early Neolithic Nea Nikomedeia, Greece. Acta Botanica Neerlandica, 20, (5) : 524-538.

Vatin, C., 1962. Une inscription inédite de Macédoine. Bulletin de Correspondance Hellénique, 86 : 57-63.

Ventris, M., Chadwick, J., 1973. Documents in Mycenaean Greek, 2e ed. Cambridge University Press, Cambridge.

Willerding, U., 1973. Bronzezeitliche Pflanzenreste aus Iria und Synoro. In U. Jantzen (dir.). Tiryns: Forschungen Und Berichte, Band VI. Philipp von Zabern, Mayence, 221-240. 